\title{
Metrische Akklamationen der Byzantiner.
}

\section{Literatur.}

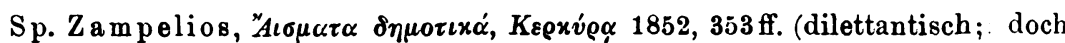
sind z. B. die Verse von III 3 richtig abgesetzt).

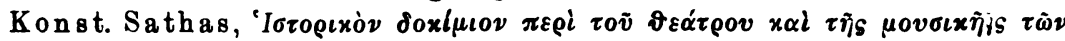
$B v \zeta \alpha \nu \tau \iota \nu \tilde{\omega} \nu$, Venedig 1878 (ein unübersichtliches und durchaus phantastisches Buch, jedoch reich an Literaturnachweisen, besonders für die nicht metrischen Akkilamationen, deren Bedeutung voll gewürdigt wird); den Hinweis auf dies Werk (danke ich Herrn Prof. Polites.

Sp. Lambros, Collection de romans grecs, 1880, introd. p. $8 \mathrm{ff}$.

E. A. Sophocles, Greek Lexicon of the Roman and Byzantine Peeriods, 2. ed. 1870 (mir nicht erreichbar), 3. ed. (posthum) 1888, $50 \mathrm{f}$.

H. Wäschke, Studien zu den Ceremonien des Konstantinos Porphyrogemnetos, Festschrift des Francisceums in Zerbst zum 37. Philologentag 1884, S. 6-144 (metrische Analyse von IX 7. 2. 3. 17, die auffällig zu Sophocles stimmt, den Wäischke nicht nennt; Zusammenstellung der Nachrichten Konstantins über die Dichtcer der Parteien und die Benennungen der Jieder).

Krumbacher GBL2 $792 \mathrm{f}$.

Die folgende Sammlung enthält alle mir bekannt gewordenen „metrisichen", d. h. durch klare metrische Responsion gebundenen Akklamationen der Byzaintiner, mit Ausnahme der sechs rein literarischen des 12. Jahrhnnderts, die Launpros,

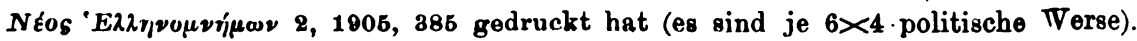
Auf einer wesentlich verschiedenen Art von Responsion beruhen die „rhythmischen" Akklamationen hellenistischen Ursprungs (Sueton, Nero 20,3), dice vom Beginn der Kaiserzeit an bei Römern und Griechen, im Zirkus wie auf dem Marsfeld, im Senat wie in der Kirche, eine gewaltige Rolle spielen (einige Litteratur bei Pauly-Wissowa 8. v. acclamatio); mit der hier behandelten Gattung thängen sie nur in Fragen der Sprache und der ẗberlieferung zusammen. Von den „,metrischen" Akklamationen der Römer (Fragm. Poet. Lat. p. 276. 330 sq. 369, 4 Baejhrens), lauter trochäischen Tetrametern, führt keine Brücke zu den byzantinischem.

\section{Zur Überlieferung.}

Von den hier mitgeteilten Texten beziehen sich I-VII amf einmalige Ereignisse, VII-X auf wiederkehrende Situationen. Dem entsprechend ist jene Gruppe von den Chronisten und Historikern, diese

1 in den Zeremonienbüchern überliefert. 
I - IV (ca. a. 532-610) stehen alle bei Theophanes; III 3 und IV 1 that auch Theophylaktos, III 1 und IV 1.2 Johannes von Antiocheïa und Kedrenos; IV 1 wird noch von einigen anderen ausgeschrieben. Da Theophanes in dem III 3 und IV 1 umschlieBenden Bericht unabhängig von Theophylaktos ist (bei dem er z. B. III 2 nicht finden kounte), aber doch manches mit ihm teilt (vgl. de Boors Randnoten), so schreiben beide dieselbe Quelle aus, die sich zwischen die Zeit der Ereignisse und die Blüte des Theophylaktos datieren läBt, also etwa 615. Das wird wohl Johannes von Antiocheia gewesen sein, dessen an Zahl so geringe Fragmente ja wirklich drei Akklamationen erhalten haben. Auf ihn dürften überhaupt alle unsere Texte zurückgehen ${ }^{11}$ ), womit erklärt wäre, warum in den Berichten der Chronisten über dlie Zeit nach 610 (IV 2) keine metrischen Akklamationen mehr zu finclen sind. - Der in unserer Handschrift des Chronikon Paschale überlieferte Anfang von I ist aus Theophanes interpoliert (vgl. Exkurs I).

Aus der Vergleichung der erhaltenen Zeugen ergibt sich über deren Qualität folgendes: Theophylaktos verdient die Glaubwürdigkeit, auf die er in beiden Fällen ausdrücklich Anspruch macht: III 3 ist durch das Metrum, IV 1 auch noch durch die Übereinstimmung mit Theophanes buchstäblich gesichert, wie er schrieb. Theophanes hat

1) Eine interessante Akklamation aus derselben Epoche, leider nicht metrisch, hat sich auf seltsame Weise erhalten. Folgendes Scholion steht gleichlautend in der. Haupths des Theophylaktos Simokattes, dem Vat. gr. 977 saec. X f. 184 (SchluB des Werkes; gleichzeitige Hand; vgl. de Boor praef. p. VI), und der Prokophs Vat. gr. 152 saec. XIV $-X V$ f. $141^{r}$ (SchluB von De bellis IV, dann eine Zeittafel der Vandalenkönige, dann das Scholion, alles von derselben Hand, die den Text der SchluBpartie des Prokoptextes geschrieben hat, Nr. 4 bei Haury prol. p. XXXIV), und ist daraus von Alemannus zu Prokop Auecd. 6, III 308 ed. Bonn und von Dindorf und Büttner-Wobst zu Zonaras XIV 12, 17 abgedruckt worden (eine genauere Beschreibung und.Kollation danke ich meinem Freund Dr. August Mayer):

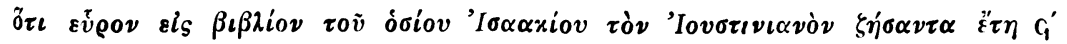

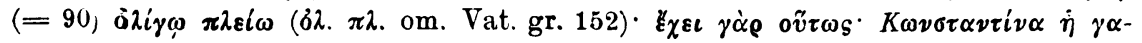

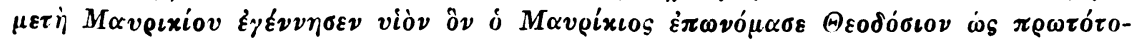

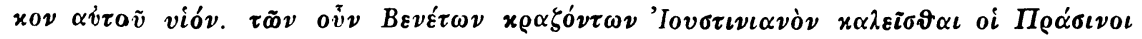

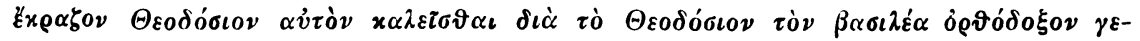

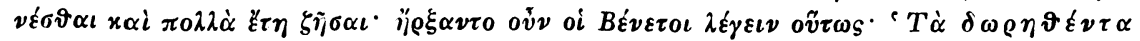

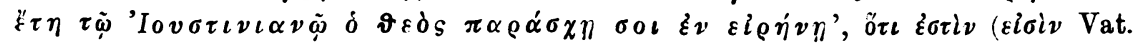

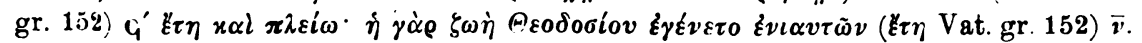

Wer der Mönch Isaakios ist, den das Scholion ausschreibt, konnte ich nicht ermitteln. Daß bei der Geburt jenes Theodosios (4. Aug. 583) des ersten „Purpurgehoruen" seit Thecdcsios II, besondere Demonstrationen geschahen, wissen wir aus Jonannes vou Ephesos, Kirchengesch. 5, 14, und aus dem SchluB des Euagrios, Hist. Eccl. 6, 24; vgl. Theophanes a. 6077 p. 254, 25. 
zwei der Stücke, wo wir ihn kontrollieren können, so stark entstellt (III 1.3), daB die Überlieferung in jenen, wo wir auf ihn allein angewiesen sind, als sehr schlecht zu gelten hat; in III 3 könnte man ohne Theophylaktos nicht einmal das Metrum finden, obwohl das in allen vier Zeilen das gleiche ist. Kein Wunder, dab wir in I, wo zu allem noch die besseren Theophaneshss fehlen, auf Schritt und Tritt straucheln. Den Johannes von Antiocheia selbst kennen wir nur durch die konstantinischen Exzerptoren, die mit seinem Text noch viel freier schalten, als die Chronisten und Historiker, wie besonders IV 1 beweist. Von den übrigen kommt für die Textkritik noch Kedrenos in Betracht, wenn er in III 1 von Theophanes unabhängig ist, was ich nicht als sicher annehmen möchte. ${ }^{1}$ )

VII. Die beiden Liedchen auf Kaiser Alexios, die seine Tochter mitteilt, stehen in der einzigen vollständigen Handschrift ihres Werkes (F) von erster Hand am Rand, bei dem der Anna gleichzeitigen Epitomator (vgl. Schopens Ausgabe praef. p. XVIII) im Text. Der Zusammenhang zeigt beidemal klar, daB Anna in ihrer Scheu vor der Volkssprache die Worte nicht ausgeschrieben hatte; von einem dritten Lied (XII cap. 6, II p. 161, 27 Reiff.) ist uns leider nur ihre Umschreibung erhalten.

IX. Konstantinos VII hatte für die Akklamationen zweifellos das beste Material, das in seiner Zeit zu haben war, nämlich die Aufzeichnungen der Parteiarchivare und Parteidichter. Und er selbst wird wohl nichts geändert haben; auch der codex unicus Lipsiensis ${ }^{2}$ ) ist gut; ein an zwei Stellen überliefertes Gedicht (5) ist buchstäblich wiederholt. Freilich zeigen zahlreiche schwere Schäden des Textes, da $B$ zwischen der Entstehung dieser Gedichte und ihrer Aufzeichnung durch die Beamten Konstantins längere Zeit verflossen war, in der man ohne Rücksicht auf das Metrum starke Umarbeitungen vornahm.

X. In jämmerlichem Zustand ist der Text des Schwalbenliedes der römischen Knaben. Der Archetypus von CV stammt von einem Schreiber, der eine nach dem Gehör mit lateinischen Buchstaben geschriebene Vorlage verständnislos und ungenau kopierte. Über die Texte, zwischen denen das Lied überliefert ist, vgl. die Ausgaben.

1) Vgl. Patzig, B. Z. IX 207. Bei den Kedrenoszitaton konnte ich eine Kollation des Marcianus cl. VII 12 (XC 5) benutzen, die mir Prof. C. de Boor freundlichst mitgeteilt hat.

2) Ich habe die Stücke nachrerglichen, ohne in Reiskes Kollation einen Fehler konstatieren zu können; Orthographica hat or mit Recht ignoriert. 
Texte.

I. Kalopodios.

OI IPA $I N O I$.

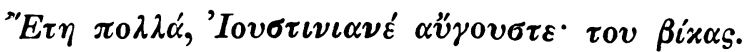

Metrum $\dot{\alpha} \delta \iota x о v ́ \mu \alpha \iota, \quad \mu \delta \nu_{\varepsilon} \dot{\alpha} \gamma \alpha \vartheta \dot{\varepsilon}^{\circ}$

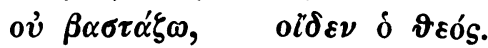

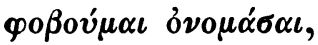

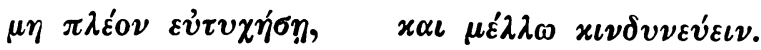

MANAATSP.

$\tau i_{S}^{\prime} \varepsilon \sigma \tau \iota \nu$, ov่x oíd $\alpha\langle\mu \varepsilon \nu\rangle$.

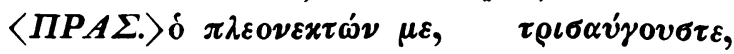

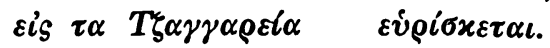

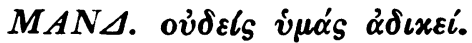

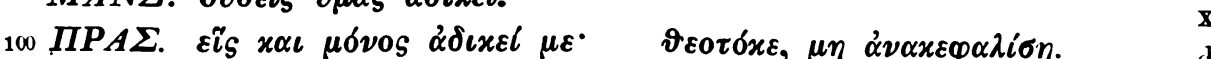

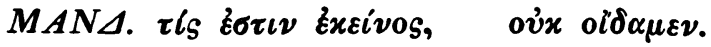

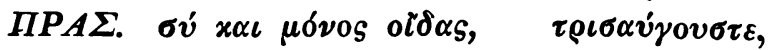

$\tau \iota \varsigma \pi \lambda \varepsilon 0 \nu \varepsilon \varkappa \tau \varepsilon l \mu \varepsilon \quad \sigma \eta \dot{\mu} \mu \rho \circ \nu$.

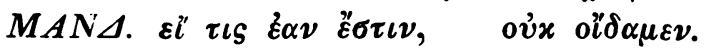

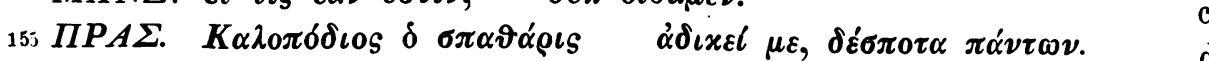

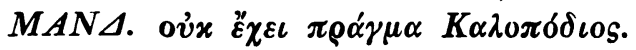

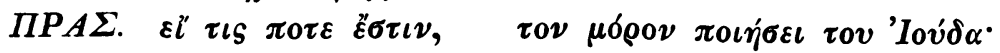

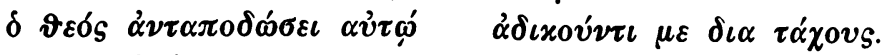

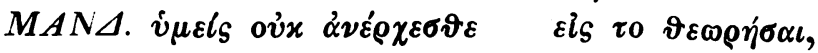

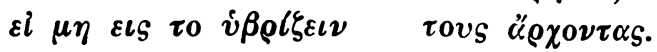

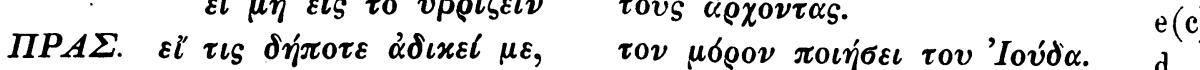

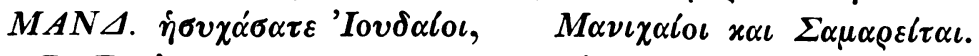

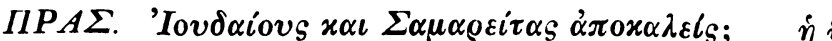

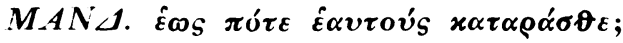

I Theophanes a. 6024, p. 181, 30-184, 1 (Hss : x y z; dazu V [vgl. Exkurs I] 1-5,

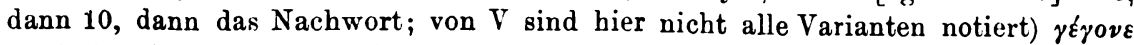

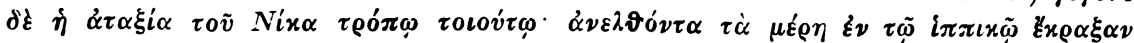

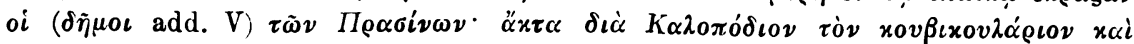

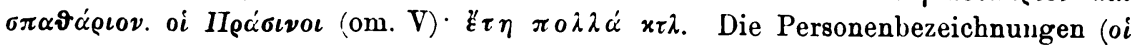

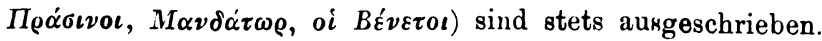

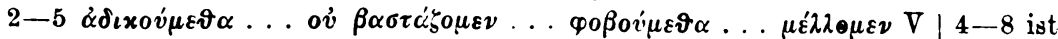

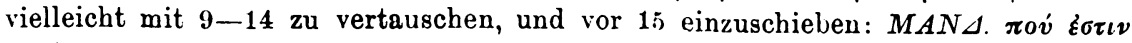

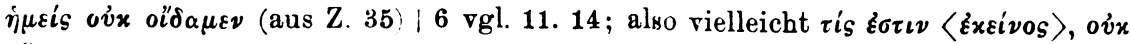

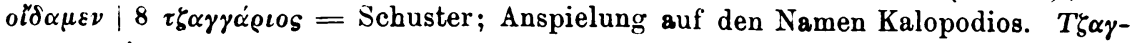

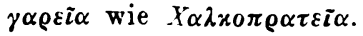

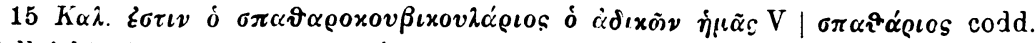
17 vielleicht $\varepsilon \check{\iota}^{\prime} \tau \iota \varsigma \delta \dot{\eta} \pi 0 \tau \varepsilon \dot{\alpha} \delta \iota x \varepsilon i \quad \mu \varepsilon$ wie 21.

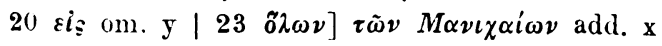




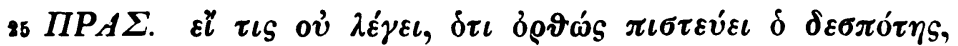
$\mathrm{xf}$

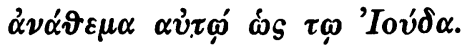

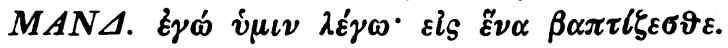

$\Pi P A \Sigma . \quad \varepsilon l_{S} \tilde{\varepsilon} \nu \alpha \beta \alpha \dot{\pi} \pi \tau \iota \xi o \mu \alpha \iota$.

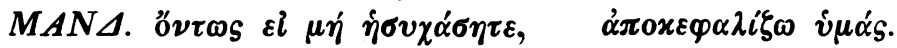

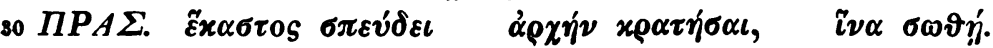

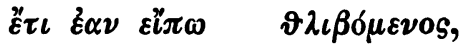

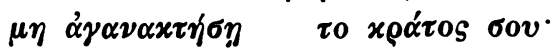

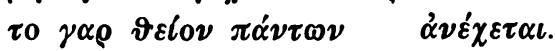

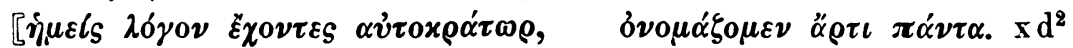

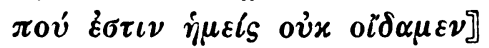

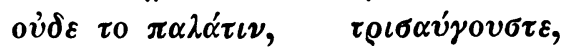

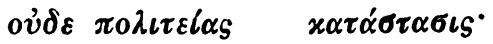

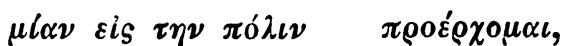

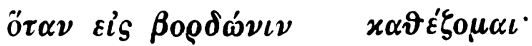

40

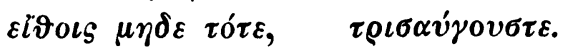

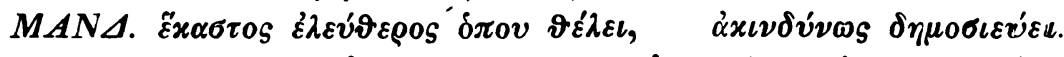

$x(c ?)$
$c$
$c$
$c$
$c$
$c$
$d(?)$
$d$
$c$
$c$
$c$
rl(?)
$d$
$g$
$g$
$d$
$h h$

45

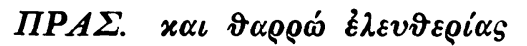
$x \alpha \iota \dot{\varepsilon} \alpha \nu\langle\tau \iota \varsigma\rangle \not ै \sigma \tau \iota \nu$.

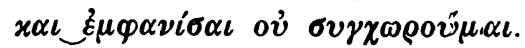

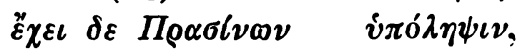

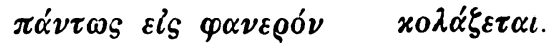

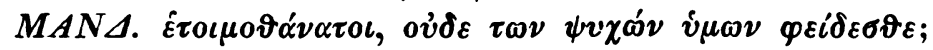

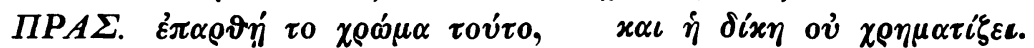

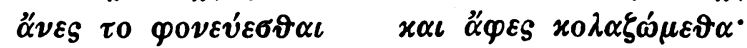

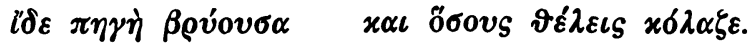

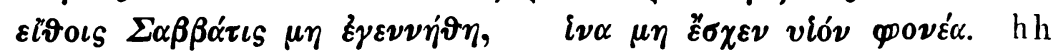

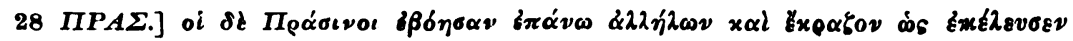

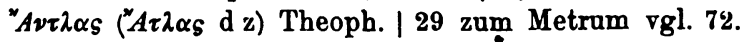

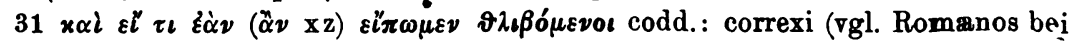

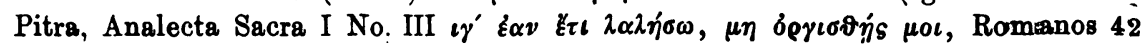

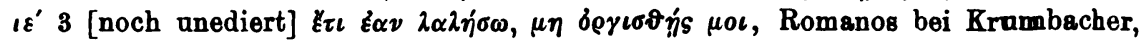
Akrostichis in der griech. Kirchenpoesie S. 668 Str. $\iota \alpha^{\prime} \varepsilon \tau \iota \tilde{\alpha} \pi \alpha \xi \xi \alpha \nu \varepsilon \varepsilon^{i} \pi \omega, \mu \eta$ beyı des MAN A. vgl. zu 4-8|36-37 vgl. Malalas in den Excerpta de insidiim 176, 14

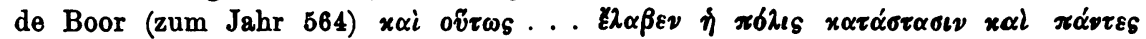

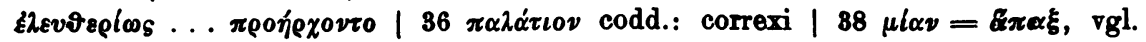

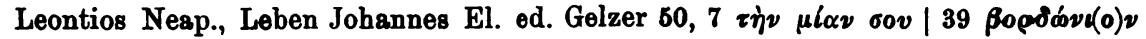
lat. burdo (vgl. Thee. ling. lat.) $=$ Maulesel. Es wird auf die Strafe der $\pi 0 \mu \pi \eta^{\prime}$ angespielt.

40 etfous viermal bei Theophanes (vgl de Boor's Index), einmal bei Romanos Pitra No. XXII $x \alpha^{\prime} \mid 47^{2}$ vgl. 76.

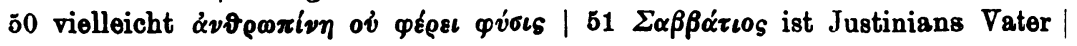




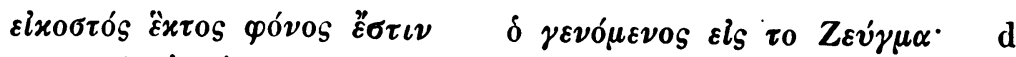

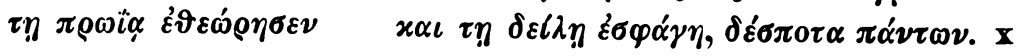

\section{OI BENETOI}

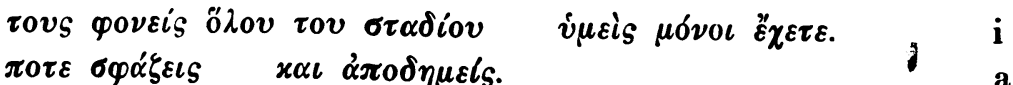

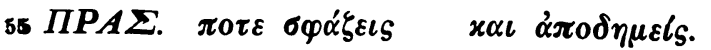

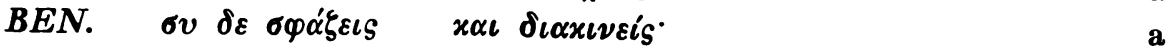

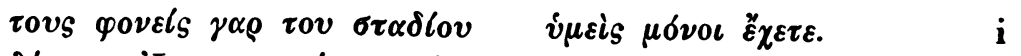

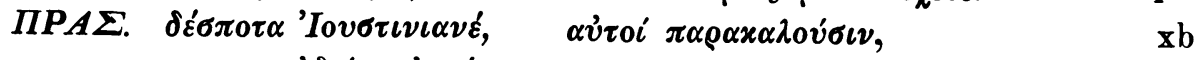

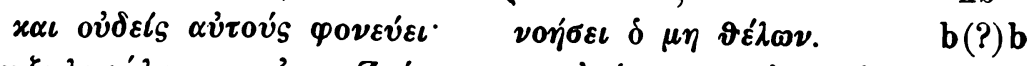

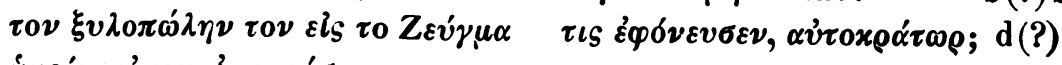

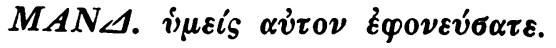

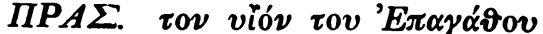

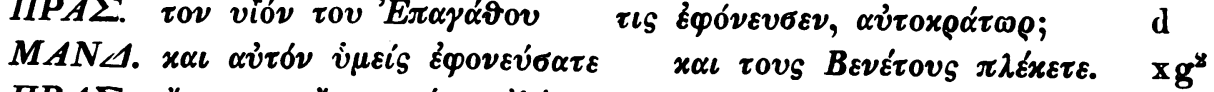

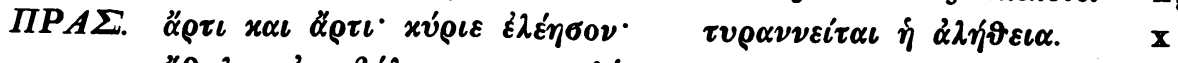

665

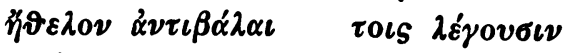

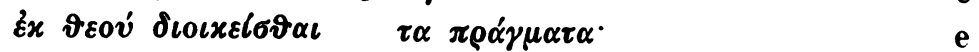
$\pi \delta \vartheta \varepsilon \nu \alpha \tilde{v} \tau \eta \dot{\eta} \delta v \sigma \tau v \chi i \alpha$

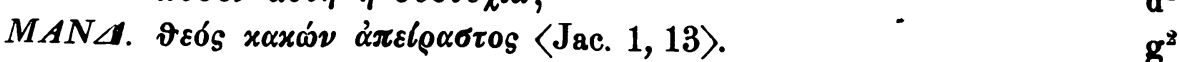

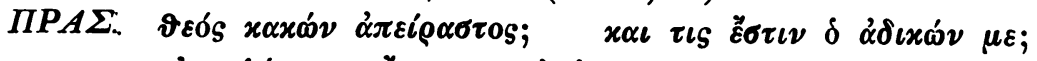

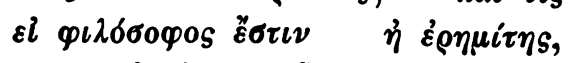

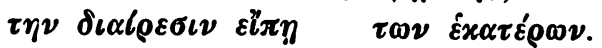

$\mathbf{x}$

$x^{8}$

e

e

$d^{8}$

$\mathbf{8}^{\mathbf{z}}$

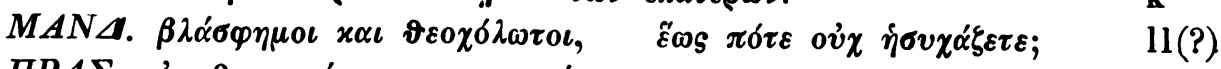

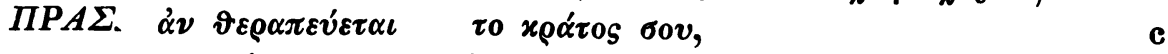

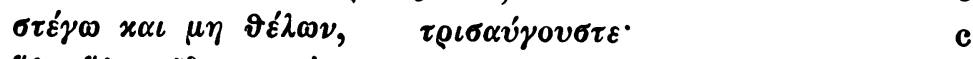

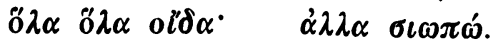

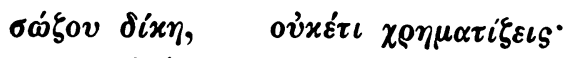

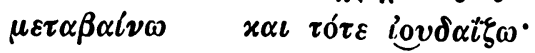

$\mu \alpha^{\prime} \lambda \lambda \sigma \nu \delta^{\prime} \dot{\varepsilon} \lambda \lambda \eta \nu i \sigma \alpha \iota \quad \sigma v \mu \varphi \varepsilon ́ \rho \varepsilon \iota \mu \iota$,

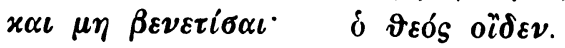

c

c

m

m

c

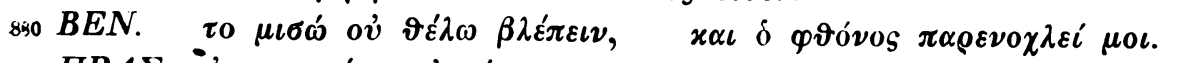

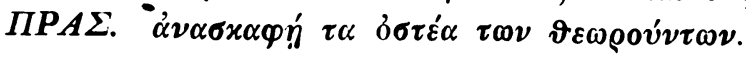

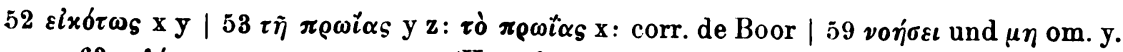
$63 \pi \lambda \varepsilon \dot{x \varepsilon l \nu}=\sigma v x \circ \varphi \alpha \nu \tau \varepsilon i \nu$ (Hesych,), $v$ gl. Leontios, vita Sym. Sal. $1718 \mathrm{C}$ $x \alpha \tau \varepsilon^{\prime} \pi \lambda \varepsilon \xi \alpha \alpha \dot{v} \tau o \tilde{v}$ und Malalas 362, 3 | 64. vgl. Const. Porphyr. De caer. 359, 7

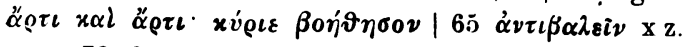

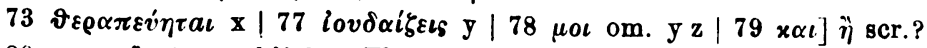

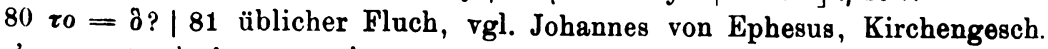

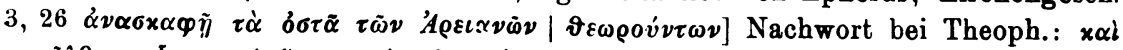

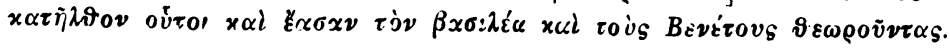




\section{Rufe der Partelen Nov. 561. zum Metrum vgl. III 1.}

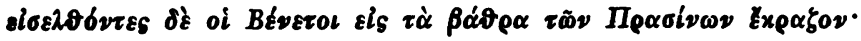

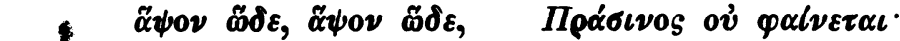

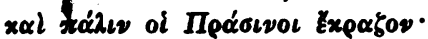

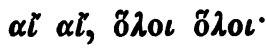

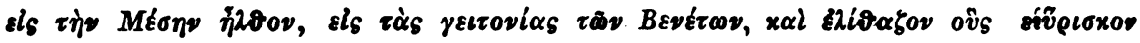

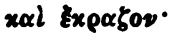

$\tilde{\alpha} \psi \circ \nu \tilde{\omega} \delta \varepsilon, \tilde{\alpha} \psi 0 \nu \tilde{\omega} \delta \varepsilon, \quad B \varepsilon \dot{v \varepsilon \tau ం \varsigma}$ ov่ $\varphi \alpha \ell \nu \varepsilon \tau \alpha \iota \cdot$

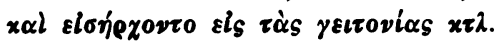

\section{II!. Rufe fïr und gegen Maurikios. \\ 1 (kurz vor November 602).}

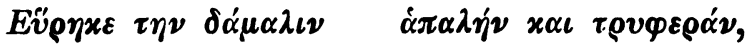

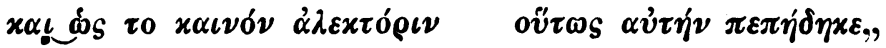

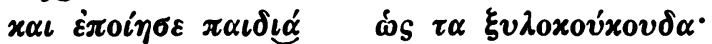

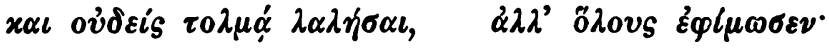

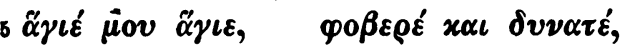

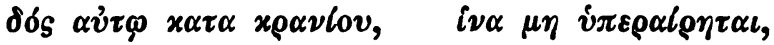

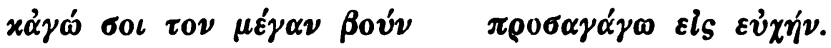

II Theophanes a. 6054, p. 236, $3 \mid 1$ zweites $\tilde{\alpha} \psi 0 \nu]$ Exat add. codd. $\mid 33$ zweites $\tilde{\alpha} \psi 0 \nu]$ Ex\&î add. codd. | zum Metrum vgl. I $54.57 \mathrm{IV} 2$ b.

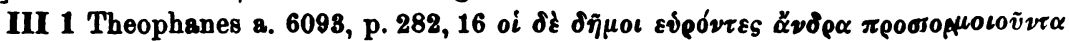

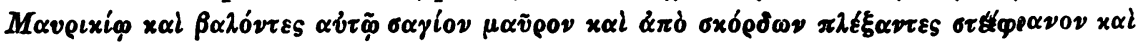

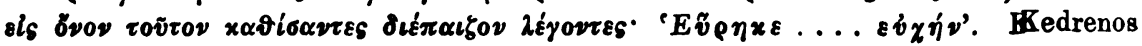

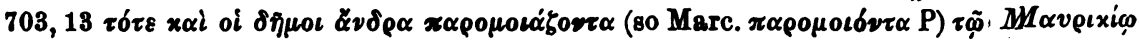

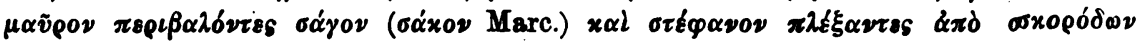

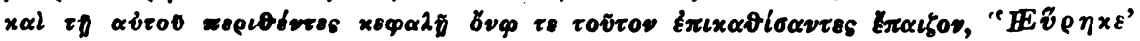

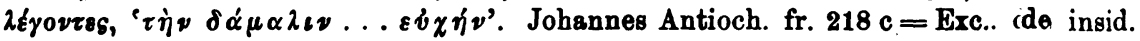

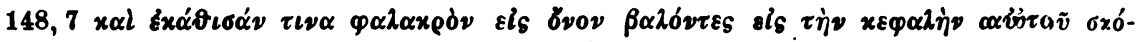

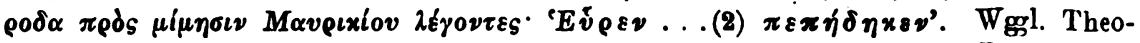
phylaktos VIII 9,8.

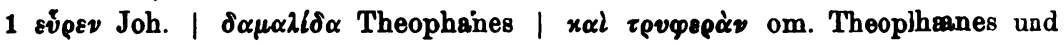

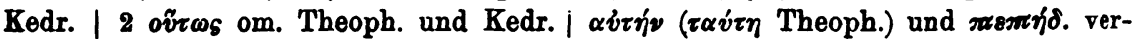

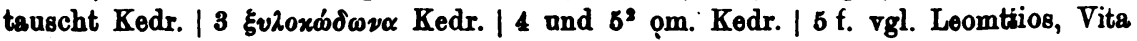

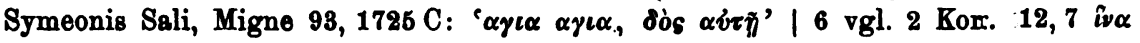

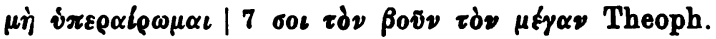

$1^{1} \cdot 3^{1} \cdot 5^{1} \cdot 7^{1}$ sind vielleicht so $\mathrm{zu}$ schreiben:

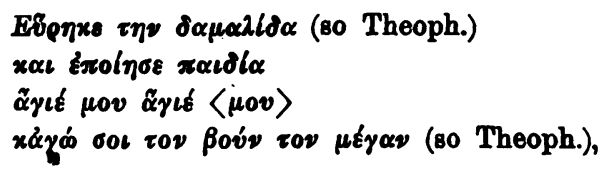

sodab alle Zeilen dasselbe Metrum hätton, nämlich das von III 2 (vgl. I i). 
2. Die Grünen.

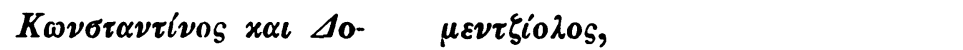

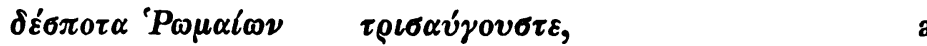

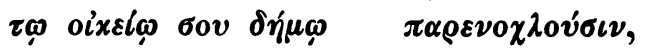

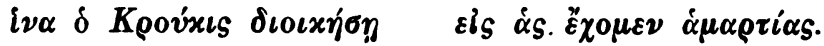

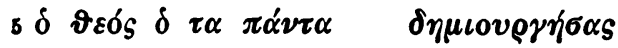

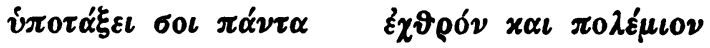

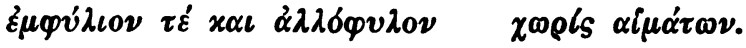

3. Die Blauen.

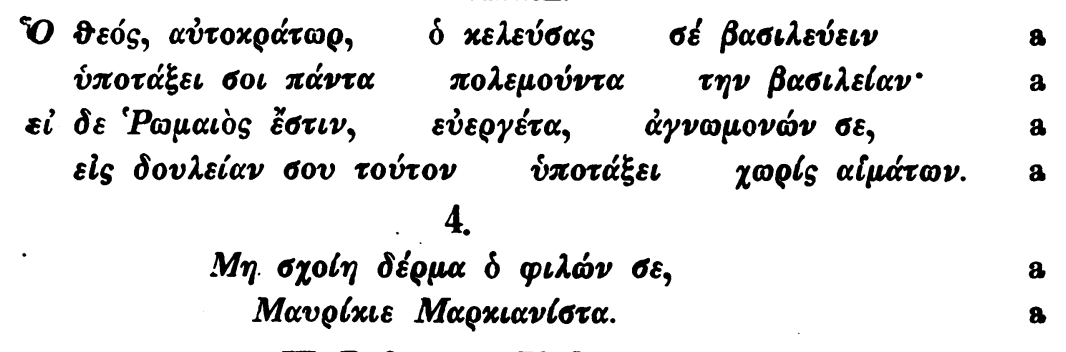

IV. Rufo gegen Phokas.

1 (26. Nov. 602). Die Blauen.

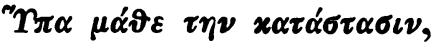

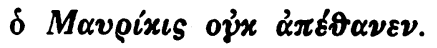

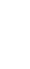


2 (a. 609/10). Die Grünen.

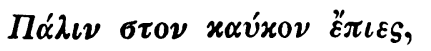

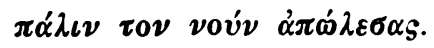

V. auf Konstantinos Kopronymos.

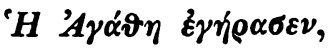

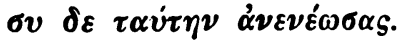

VI. auf Graf Adalbert (ca. 900).

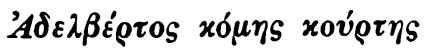

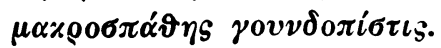

VII. auf Alexios Komnenos.

1.

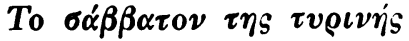

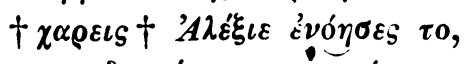

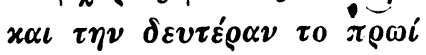

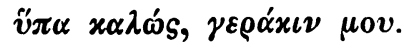

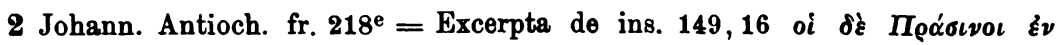

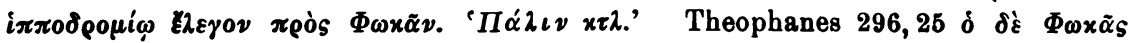

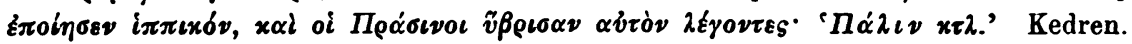

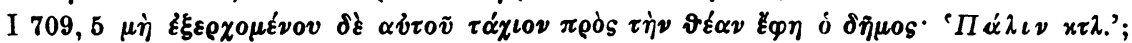

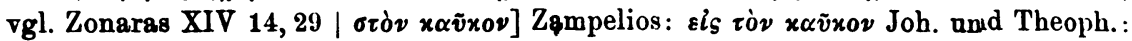

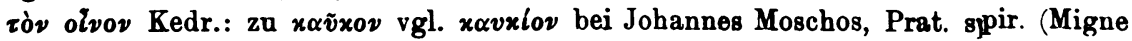

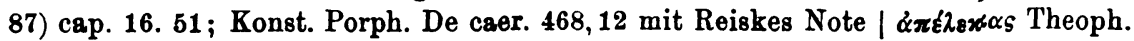

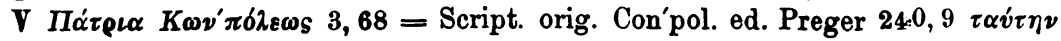

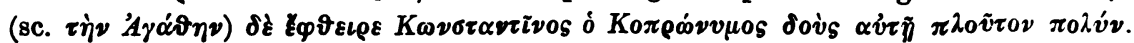

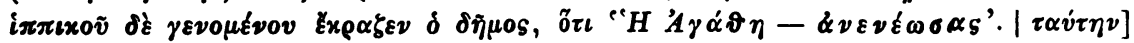
$\tau \eta \nu$ Zampelios | vielleicht \& $\nu$ Ev

VI Liudbrandi Antapodosis 2, 34 (Mon. Germ. hist. ed. Pertz V 295, 7) tam dirae autem postmodum factus est famse, ut huiusmodi vera de eo tam a maioribus quam a pueris cantio diceretur. et quia sonorius est, graece illud dicamus: A $\delta \& \lambda \beta$. $x \tau \lambda$. Adelbertos comis curtis macrospathis gundopistis, quo significatur et dicitur longo eum uti ense et minima fide $\mid$ xov́ Lambros. | Mein Kollege Ad. Hofmeister hält das Stück für Erfindung des Liutprand.

VII 1 Anna Komnene, Alex. II cap. 4, I p. 68, 29 Reiff. (Alexios hat sich durch

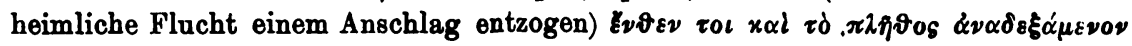

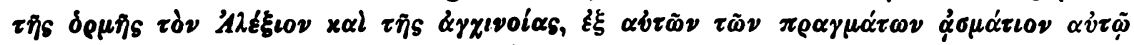

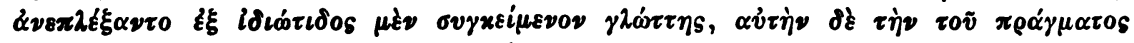

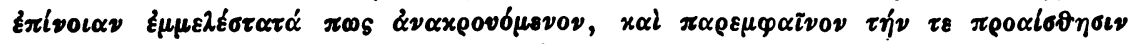

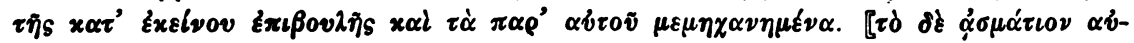

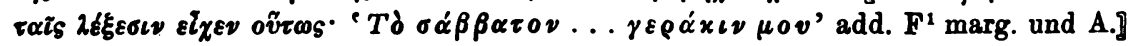

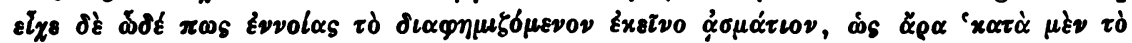

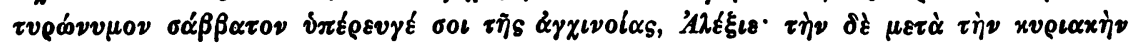

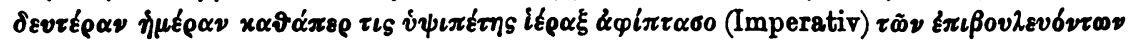
$\beta \alpha \rho \beta \alpha \dot{\rho} \rho \omega$ | vgl. Papadimitriu in der von Kurtz, B. Z. II 141 exzerpierten Publikation | $4 \tilde{v} \pi \alpha$ vgl. IV 1; $\tilde{v} \pi \alpha \gamma \varepsilon x \alpha \lambda \bar{\omega} \varsigma$ Leontios, Vita Symeonis Sali, Migne 93, 1717 A. 
2.

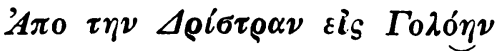

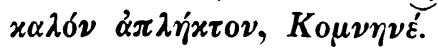

VIII. Auf den Pagarchen von Arsinoe.

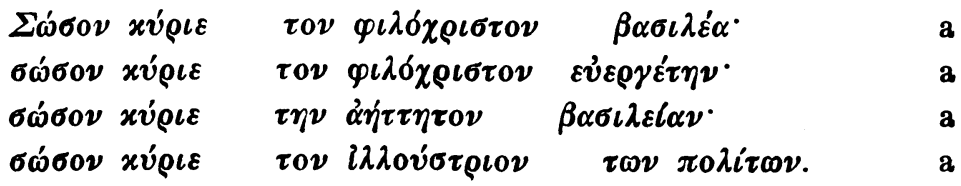

\section{Hleichzeilige und antistrophische Lieder bei Konstantinos Porphyrogen- netos, De caerimoniis.}

1. Politische Verse.

\begin{tabular}{|c|c|}
\hline 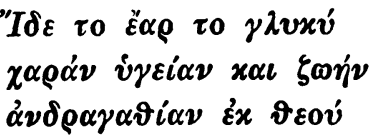 & 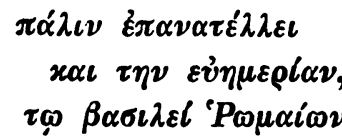 \\
\hline & \\
\hline
\end{tabular}

Metrum von 2-4: Alternierende proparoxytonische Acht- und Siebensilber, distichisch. Vereinzelte Verse desselben MaBes 264, 22. 351, 13. 357, 13.

\section{Zur Hochzeit des Kaisers.}

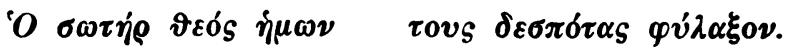

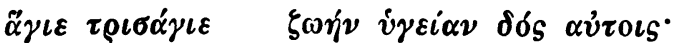

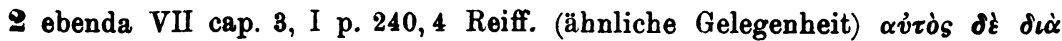

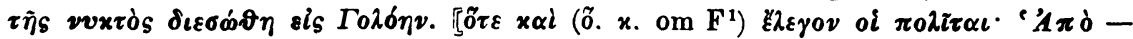

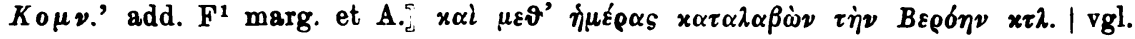
Papadimitriu l. c. (zu 1) $\mid \dot{\alpha} \pi \lambda \dot{\eta} \dot{x} \tau o \nu=$ Reise.

VIII Pap. Ber. 5603 saec. VI-VII ed. Krebs, Göttinger Nachrichten 1892 Nr. 4, II. Vorher gehen, durch einen Strich getrennt, unmetrische Akklamationen

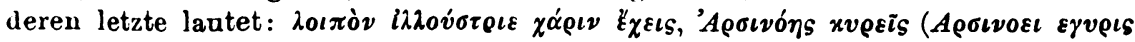

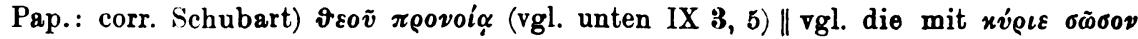
beginnenden unmetrischen Akklamationen bei Theophanes a. 6024, p. 185, 14; Konst. Porph. de caer. 279. 282. 315. 321. 331. 356. 359. 368; außerdem Theophanes

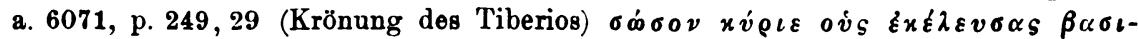

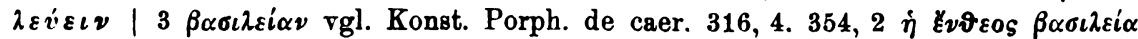

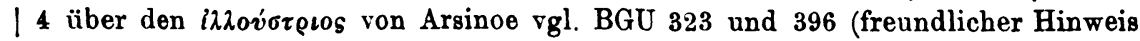
von Wilh. Schubart) | $\pi 0 \lambda i \tau \omega \nu$ drei- und mehrsilbige $-\alpha$ Stämme haben bei den Byzantinern oft paroxytonischen Genetiv des Plural.

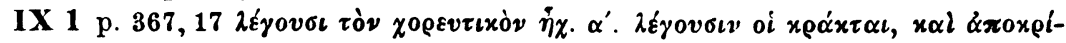

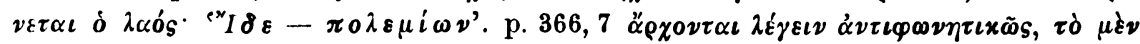

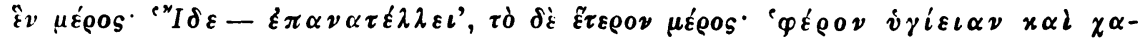

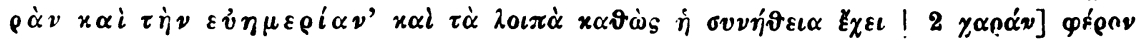
p. $\left.366 \mid \zeta \omega \eta^{\prime} v\right] \chi \alpha \rho \alpha \dot{v}$ p. $366 \mid 3$ roĩ $\beta \alpha \sigma \iota \lambda \varepsilon \tilde{v} \sigma \iota$ cod.: corr. Krumbacher GBL 255.

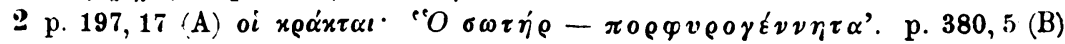




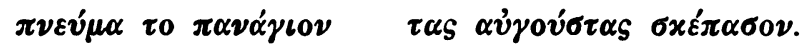

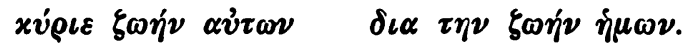

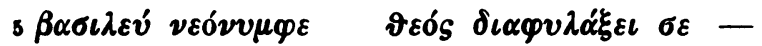

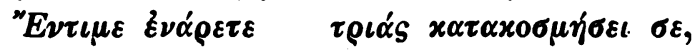

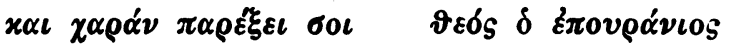

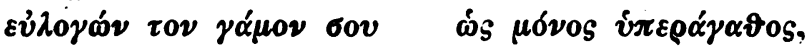

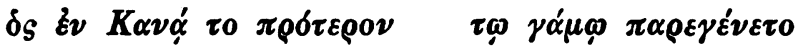

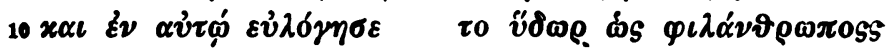

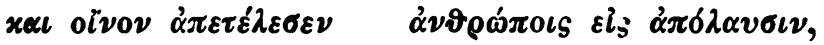

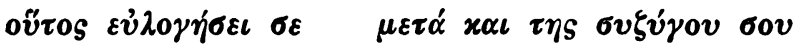

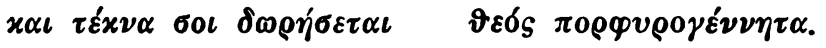

3. BegrüBung des Demarchen.

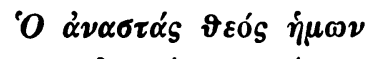

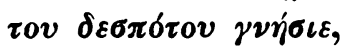
$x \alpha \iota \pi \lambda \varepsilon i 0 \nu \alpha \delta \omega \varrho \eta \sigma \sigma \tau \alpha \iota$

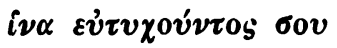

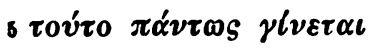

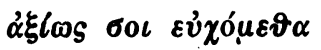
$\delta \tau \iota$ i $\pi \varepsilon \rho \beta \alpha \dot{\lambda \lambda o v \sigma \alpha \nu}$

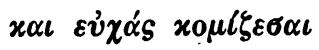

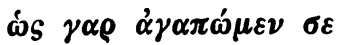

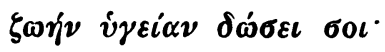

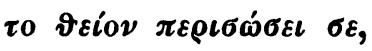

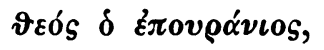

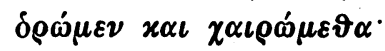

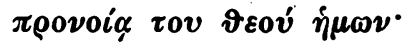

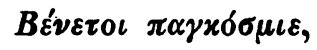

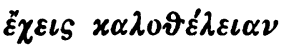
$\varepsilon x \tau \omega \nu \stackrel{\alpha}{\alpha} \alpha \pi \dot{\tau} \omega \tau \omega \nu \quad \sigma \varepsilon$.

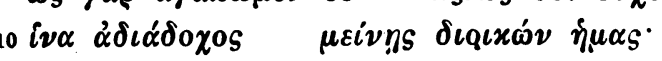

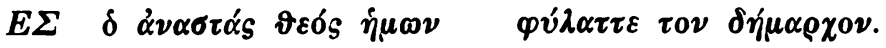

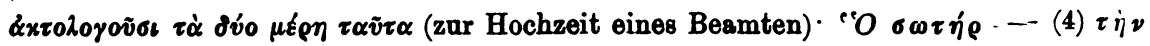

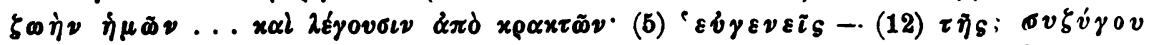

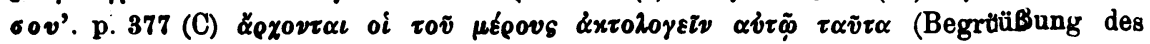

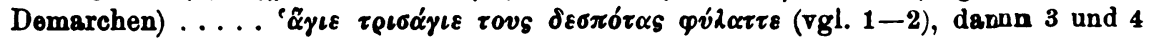
bis $\tau \dot{\eta} \nu \zeta \omega \dot{\eta} \nu \hat{\eta} \mu \tilde{\omega} \nu$.

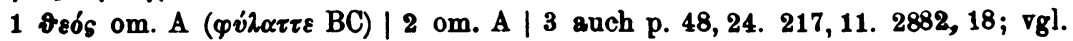

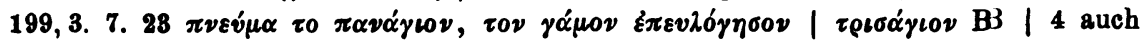

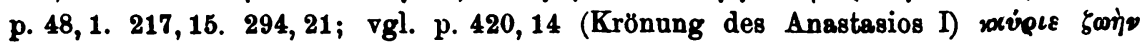

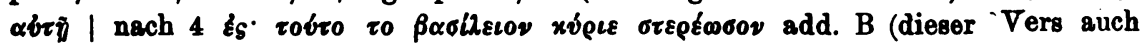

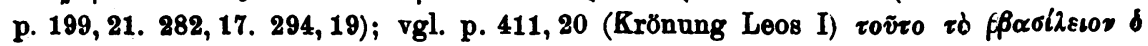

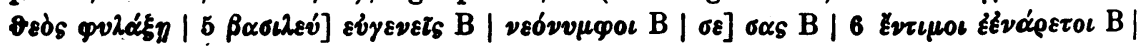

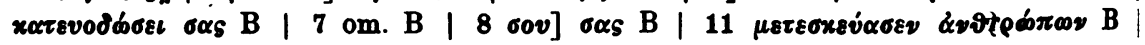

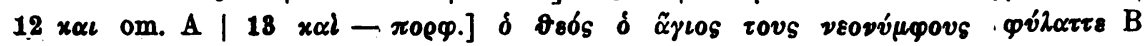

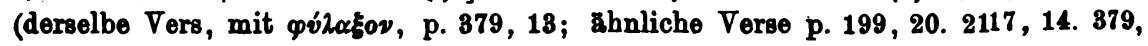
18. 14).

8 p. 377, 17 als Sohluß einer längeren Reihe von Rufen (vgl. zu 2$) 16^{1}$ und $6^{8}$ wohl zu vertauschen (vgl. 4, 9) | 9 os und ool vertauscht cod. | 11 vgll. p. 378, 21

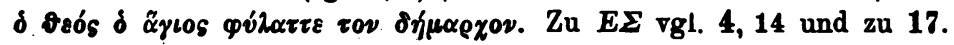


4. Auf den Stadtpräfekts

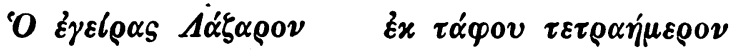

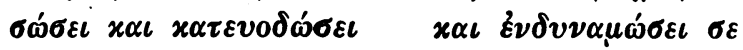

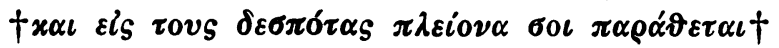

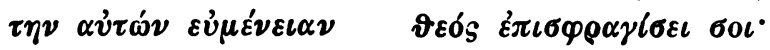

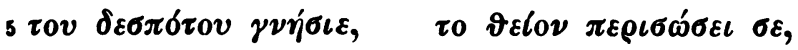

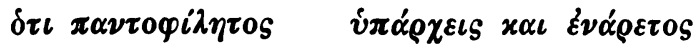

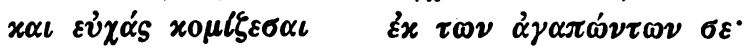

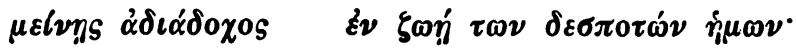

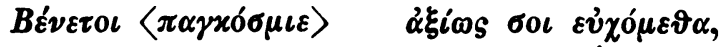

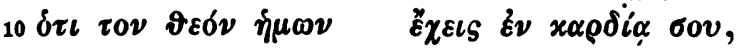

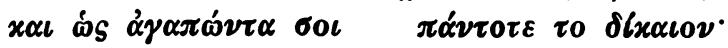

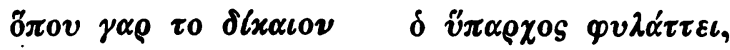

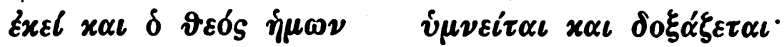

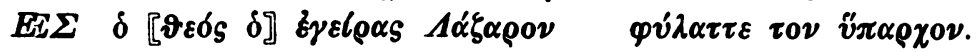

Meetrum ron 5-12: paroxytonische Achtsilber, distichisch; jedes Gedicht besteht aun acht anch ihrerseits distichisch gegliederten Langzeilen. Am Halbzeilensichluß $B$ ist auch proparoxytonischer SchluBakzent gestattet.

\section{Zur Krönung.}

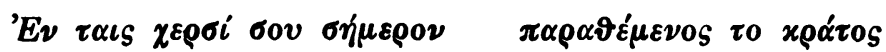

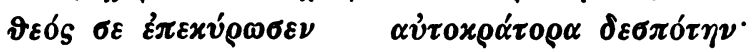

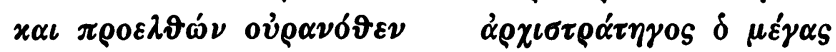

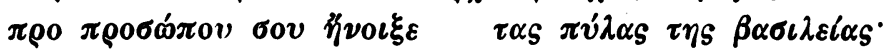
t5

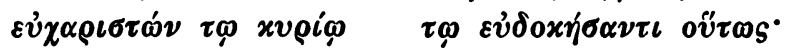

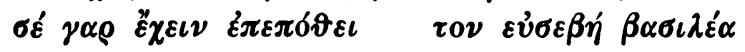

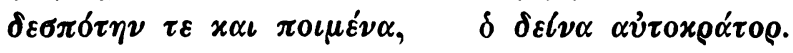

6. Zum Natalis imperii.

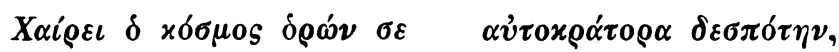

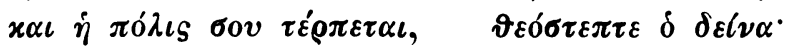
$\dot{\omega} \varrho \alpha i \zeta \varepsilon \tau \alpha \iota \quad \dot{\eta} \tau \alpha^{\prime} \xi \iota \varsigma \quad \sigma \varepsilon \beta \lambda \dot{\varepsilon} \pi 0 v \sigma \alpha \quad \tau \alpha \xi\llcorner\alpha \dot{\rho} \propto \eta \nu$,

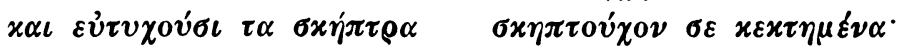

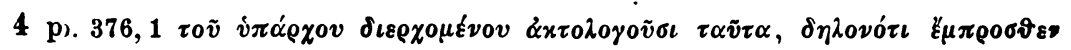

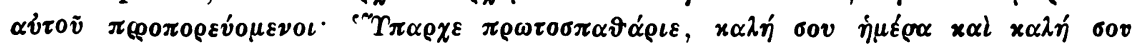

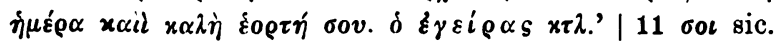

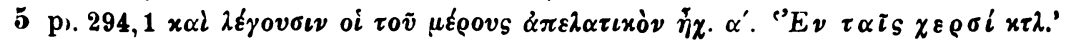

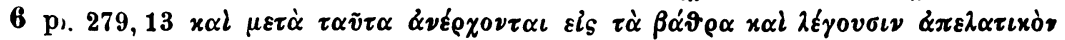

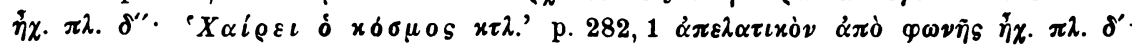

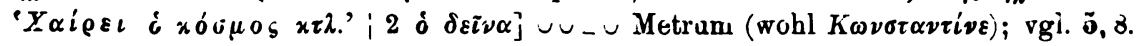
$8,2 \mid 3 \dot{\eta} \tau \alpha^{\prime} \xi \iota s$ ist der Senat (vgl. Reiske) $\mid 7$ vgl. 8, 7. 


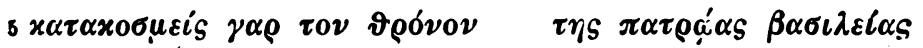

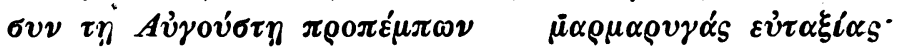

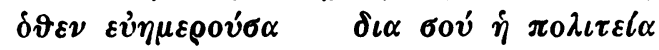

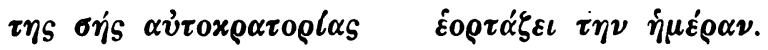

7. Hochzeitslied.

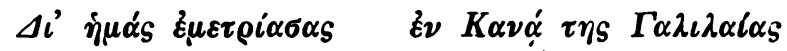

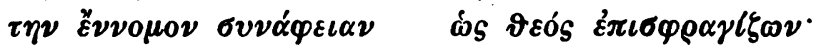

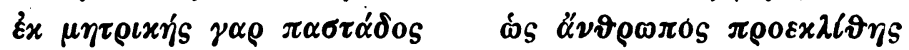
$\langle x \times \times \times \times \times \times v \quad x \times x \times x \times-v$ $5 \times x \times x \times x \times v \quad \times x \times x \times x-v\rangle$

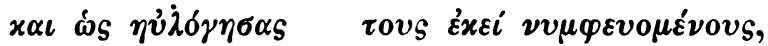

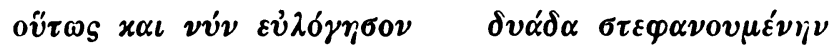

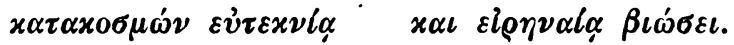

8. Begrüßung des Kaisers.

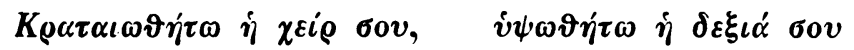

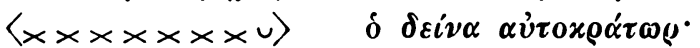

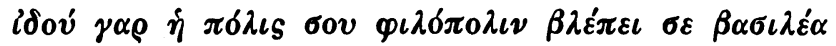

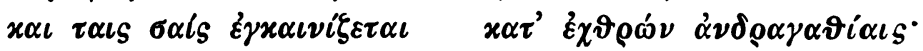

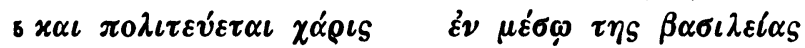

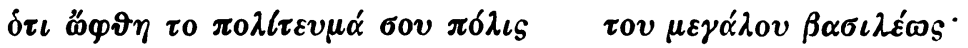

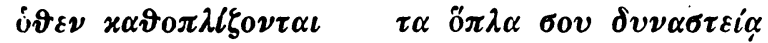

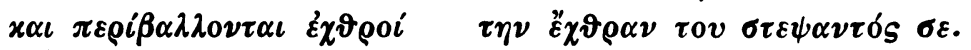

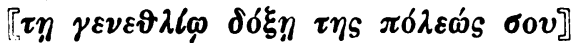

9. BegrüBung des Kaisers.

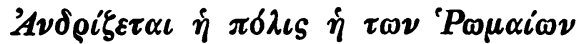

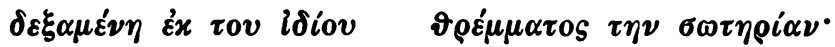

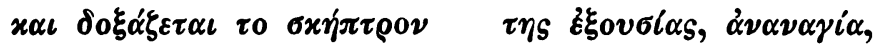

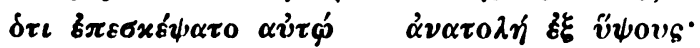

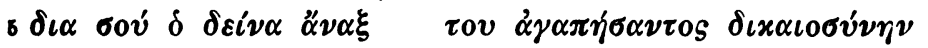

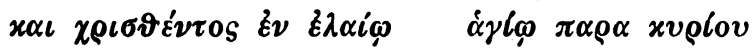

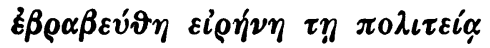

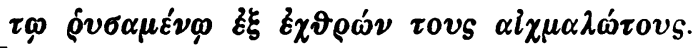

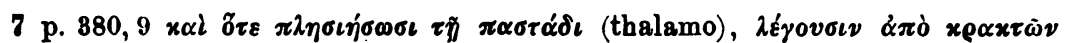

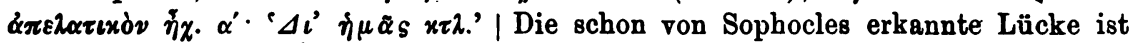
nicht sicher zu lokalisieren.

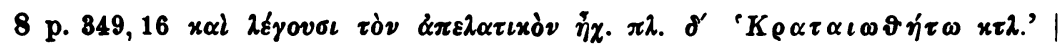

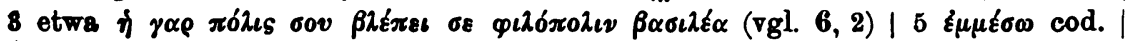

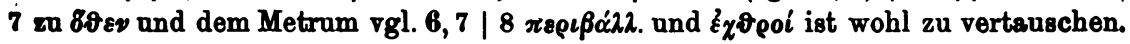

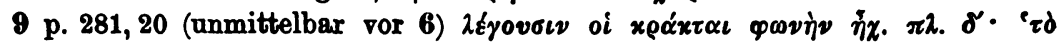

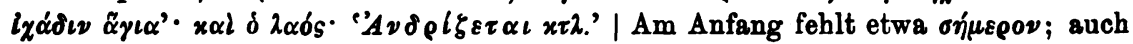
der Rest ist schwer verdorben | 8 \& $\alpha \alpha \nu \alpha j i \alpha$ vgl. zu XI 17. 
10-15. Osterlieder.

10.

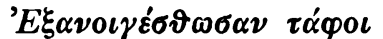

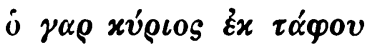
$\varepsilon \dot{v} \varphi \varrho \alpha \iota \nu \varepsilon ์ \sigma \vartheta \omega \sigma \alpha \nu$ oi $\xi \omega \dot{\nu \tau \varepsilon \dot{~}}$

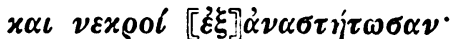
$\sigma \eta_{\mu \varepsilon \rho \circ \nu} \dot{\varepsilon} \xi \alpha \nu \varepsilon^{\prime} \sigma \tau \eta^{\circ}$

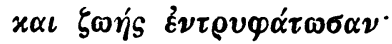

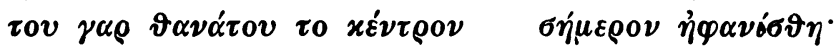

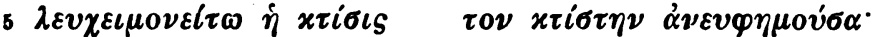

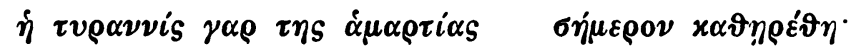

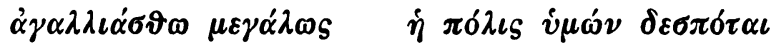

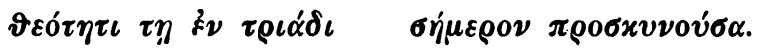

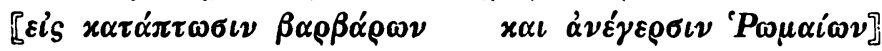

11.

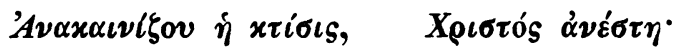

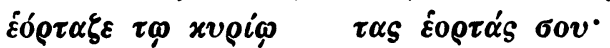

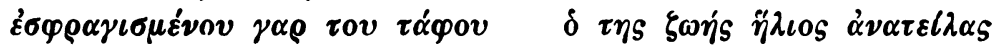

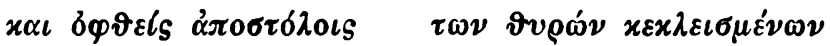

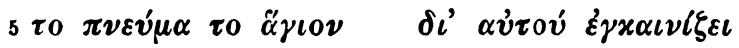

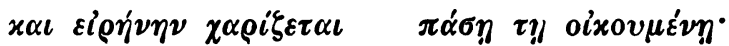

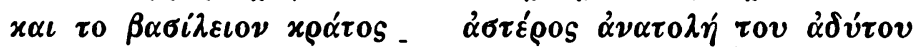

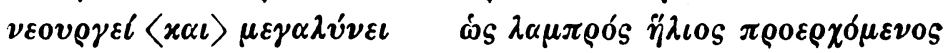

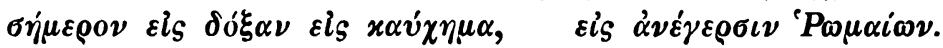

12.

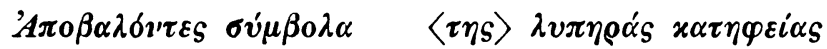

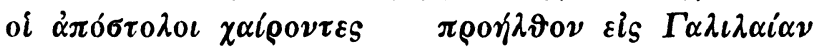

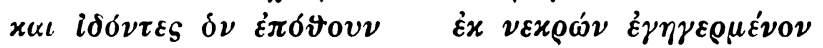

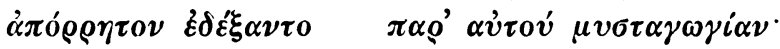

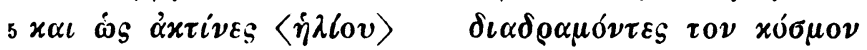

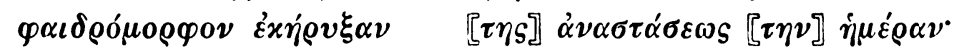
$\alpha \dot{v} \tau \omega \dot{\nu}\langle\nu v \nu\rangle \dot{\eta} \pi \rho 0 \sigma \tau \dot{\tau} \sigma \dot{i} \alpha$ $\tau \eta \vartheta \boldsymbol{\vartheta} \varepsilon i x \eta \dot{~} \pi \alpha \nu \tau \varepsilon v \chi i \alpha$

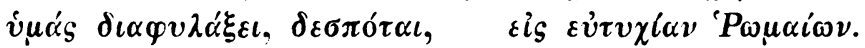

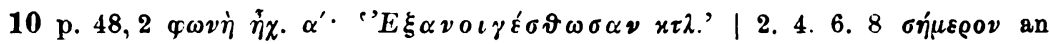
derselben Versstelle | $6 \dot{\alpha} \mu \alpha \rho \tau i \alpha s]$ etwa $\pi \lambda \dot{\nu} \nu \eta s$ scr.

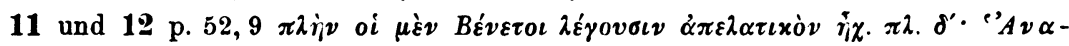

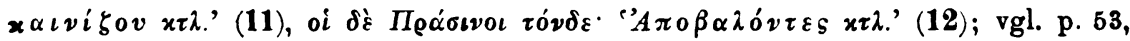
7-8, wo die Anfänge von 11 und 12 zitiert werden, ietzterer mit der Variante

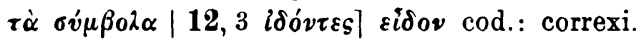




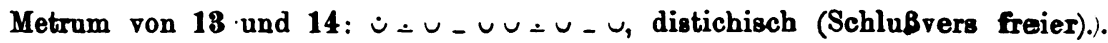
13.

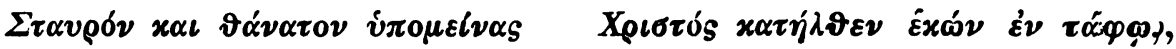

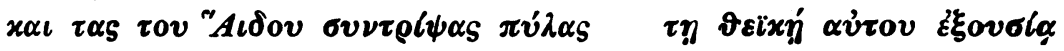

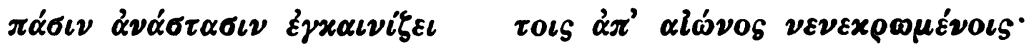

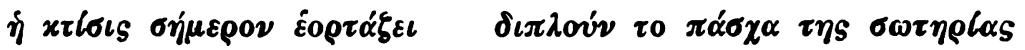

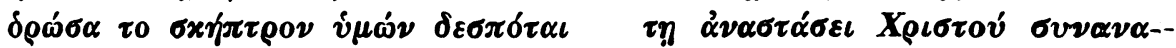
$\tau \varepsilon \dot{\lambda \lambda \partial \nu . ~}$

\section{4.}

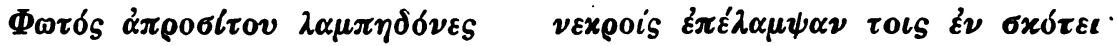

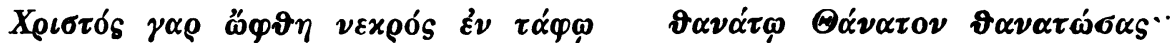

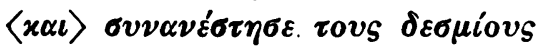

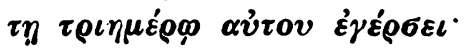

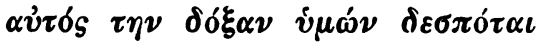

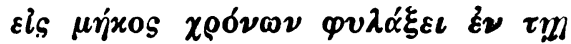

15.

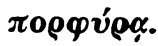

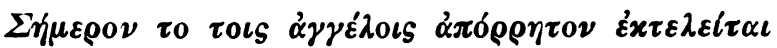

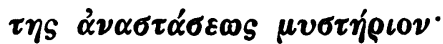

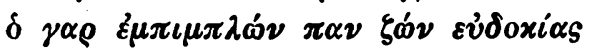

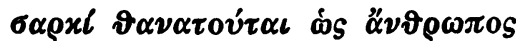

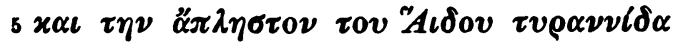
a

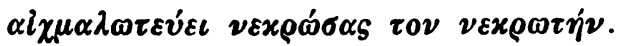

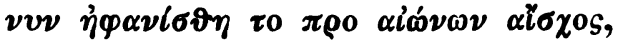

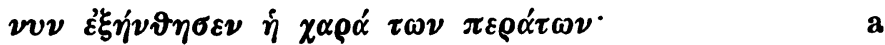

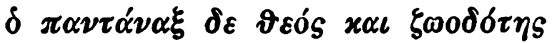

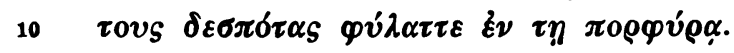

16. Zur Theophanie.

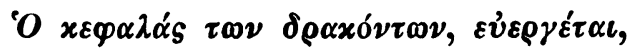
a

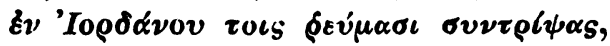

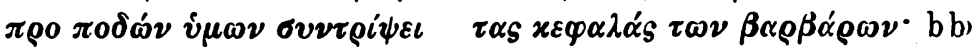

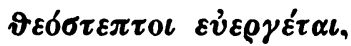

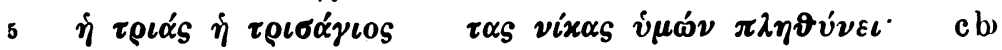

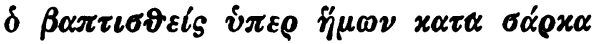

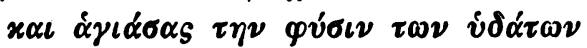
$\langle\ldots \ldots . \ldots . . . . .$.

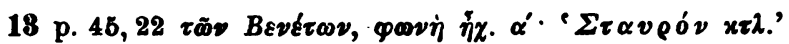

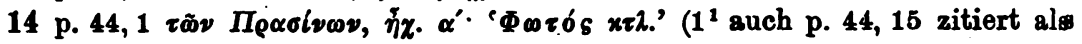

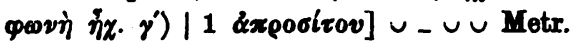

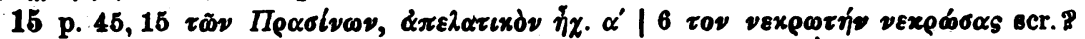

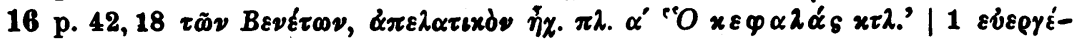
$\tau \alpha \iota]$ vgl. III 4, 3. 


\section{Alphabetarion.}

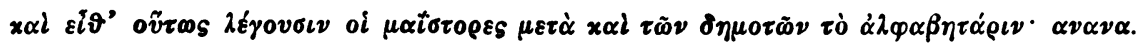

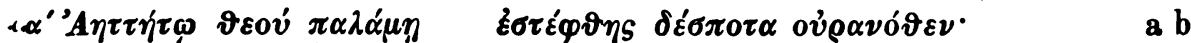

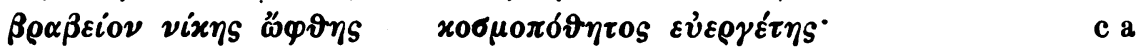

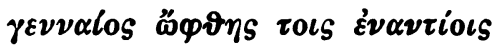

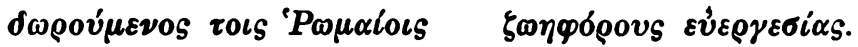

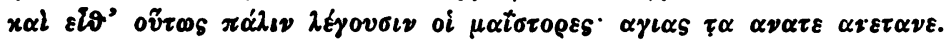

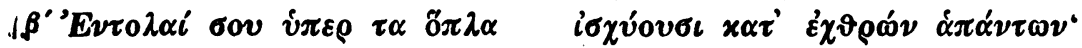

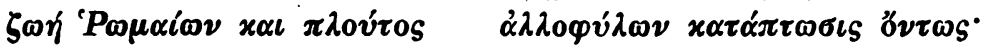

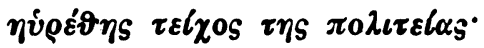

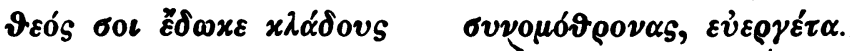

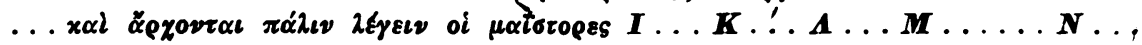

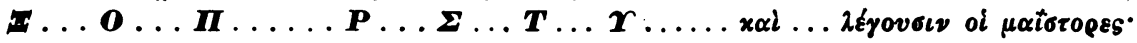

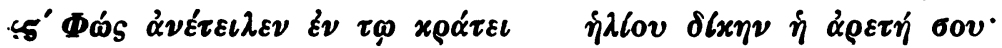

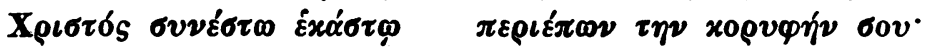

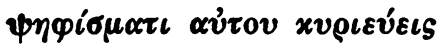

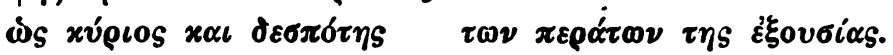

\section{Chelidonisma der römischen Knaben.}

Metrum: Freie Siebensilber (paroxytonischer SchluB streckenweise vermieden), distichisch. Dasselbe Metrum haben neugriechische Schwalbenlieder (bei Passow).

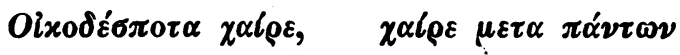

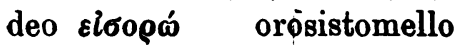

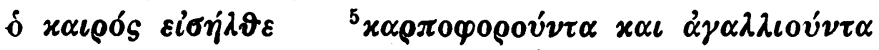

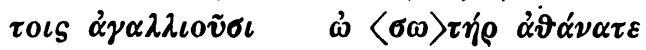

17 p. 383, $4 \| \alpha^{\prime}$ Ưberschrift $\alpha \nu \alpha \nu \alpha$ und $\beta^{\prime}$ toberschrift $\alpha \gamma \iota \alpha s \quad x \tau \lambda$.] über diese

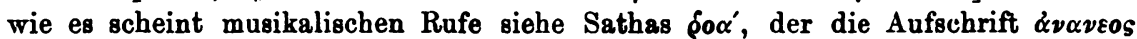
u. ähnl. auf Münzen des 7. Jahrh. vergleicht $\| \alpha^{\prime}-5^{\prime}$ in der Anrede ist überall

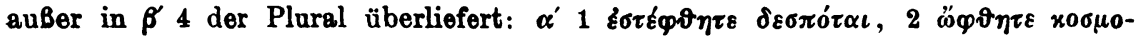

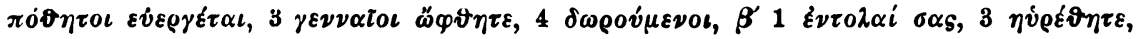

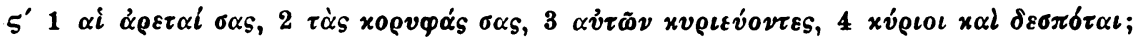

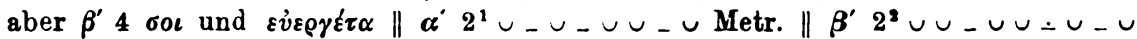

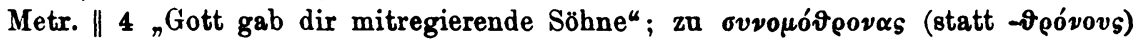
vgl. Reiskes Note; wenn nicht auch hier der Plural interpoliert ist, so kann nur Basileios I gemeint sein.

- $\quad X$ Überlieferung: $\mathrm{C}=$ cod. Cambrai 512 saec. XII

$\mathrm{V}=$ Vallic. F 73 saec. XV.

Beide Hss geben den Text in lateinischer Transkription; eine sorgfältige Abschrift druckte Tommasini (s. u.). Orthographische Varfanten (auch $\mathrm{k}=\mathrm{ch}, \mathrm{t}=\mathrm{th}$ ) und Sonderlesarten von $\mathrm{C}$ oder $\mathrm{V}$, wo der Text feststeht, sind hier nicht notiert.

ed. Fabre, Le polyptyque du chanoine Benoît, Lille', 1889 (GBL $\left.256^{4}\right)$ und V. Tommasini in 'Scritti vari di filologia, à Ernesto Monaci', Rom 1901, 382.

1-14 Langzeilenteilung unsıcher $\mid 2 \mu \varepsilon \tau \alpha]$ meto $\mathrm{CV} \mid 3$ oros] or $\mathrm{V} \mid 4$ ceras $\mathrm{C}$ : cheras V: corr. Fabre $\left.\mid \begin{array}{ll}6 & \tau o c\end{array}\right]$ tif $V \mid\langle\sigma \omega\rangle \tau \eta \dot{\rho}$ Tommasini: tyr C: thityr V: auch 


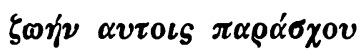
$\pi \varrho \delta ́ \beta \alpha \tau \alpha \quad \pi \tau \eta \nu \sigma^{\prime} \pi 0 v \lambda \alpha$ timisuntes colites

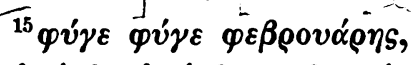

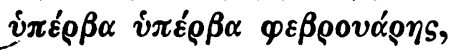

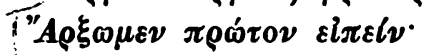

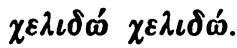

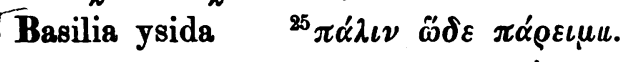

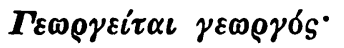
$\Delta \iota \alpha \tilde{v} \delta \omega \rho \quad x \alpha \iota \pi \eta \lambda \boldsymbol{v}^{\prime}$

${ }^{30} \dot{\alpha} \beta \eta \dot{\eta} \nu \alpha \quad \dot{\alpha} \beta \eta^{\prime} \nu \alpha$ via via $\mu \eta \dot{\eta} \mu \varepsilon \delta \varepsilon l \varrho \eta \mathrm{S}$ $\pi \dot{\nu} \nu \varepsilon \varepsilon \varepsilon^{\prime} \nu \tau \varepsilon$,

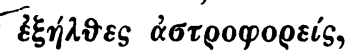

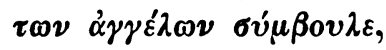

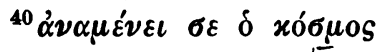
$\dot{\alpha} \gamma \alpha \lambda \lambda\llcorner\alpha \dot{\sigma} \sigma \vartheta \varepsilon \pi \alpha \ell \delta \varepsilon S$

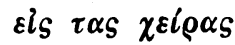
${ }^{25}{ }_{\alpha}^{\prime} \lambda \lambda \alpha \pi \varepsilon^{\prime} \nu \tau \varepsilon$, $\varkappa \alpha \tau \alpha \pi \dot{\alpha} \nu \tau \alpha \quad \varepsilon \dot{v} \vartheta v \mu \varepsilon l$.

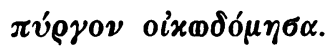

et kelegasi

${ }^{10}$ singinuntta $\tau \varepsilon ́ x \nu \alpha$ damarin tinagria oschemús manthanone

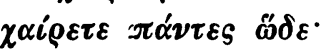

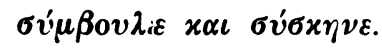

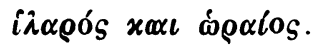

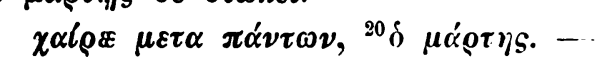

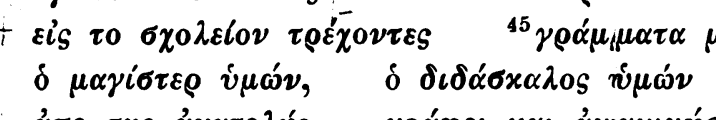

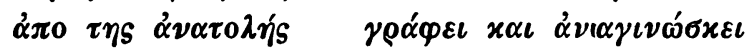

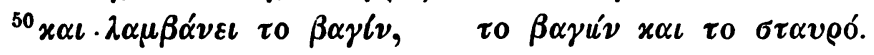

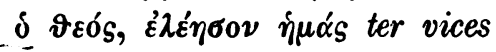

हैं

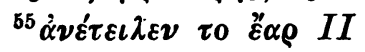

$\delta \iota \alpha$ боv $\xi \eta^{\prime} \tau \alpha \pi \alpha^{\prime} \nu \tau \alpha I I$

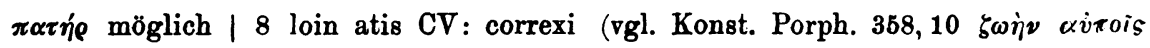
$\pi \alpha \rho \alpha ́ \sigma x o v)$.

$11 \pi \tau \eta v o ́ \pi 0 v \lambda \alpha$ ] tinabula CV: correxitt Krumbacher B. Z. XI 587 ( $\pi \tau \eta \nu \alpha^{\prime}, \pi 0^{i} \cdot \lambda \alpha$

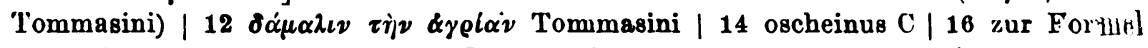

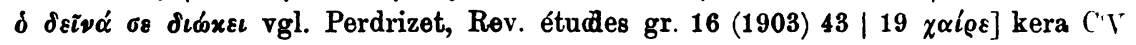
15. 17 februarie CV (vgl. 53).

$21 \mathrm{ff}$. Akrostichis von Orphanides erkannt | 24 ysida $\left(\varepsilon{ }^{\prime \prime} \sigma \varepsilon \iota \delta \alpha\right)$ C: adisa $V$ 25 parum C: parinu V: correxi | 27 etttini C: bthim V: corr. Orphanides (vgl.

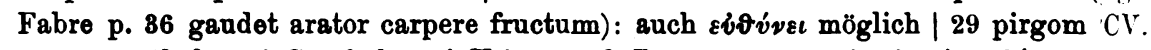

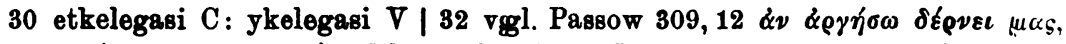

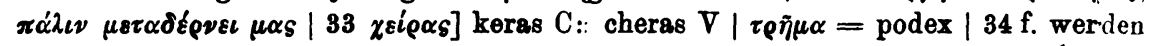
die Schläge gezählt oder die Eier, die dïe Kinder bekommen? vgl. Passow 309, 8

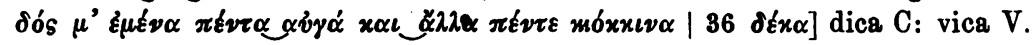

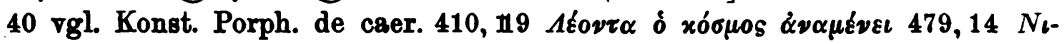

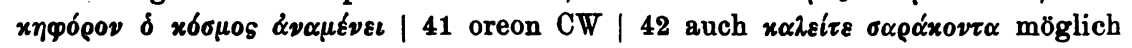

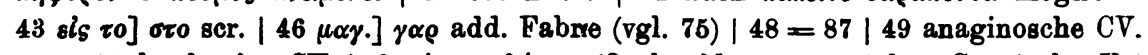

50 lambanim CV $\mid \beta \alpha y i \nu=\beta \alpha$ íiov (Orphanides) $\mid 51$ stobro C: strobo V | 56-59 om. V | 56 biyazusitubanda II. (sic) C: correxi. 
diadadascale $\delta$ จ $\varepsilon \delta_{S} \sigma \varepsilon \varphi v \lambda \alpha \dot{\xi} \eta$ III

${ }^{60}$ filoponuntus $\mu \alpha \vartheta \eta \tau \alpha \dot{s}$ бov II

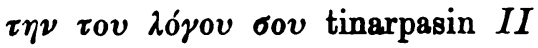

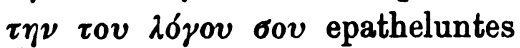

'P

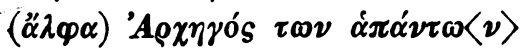

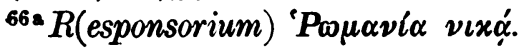

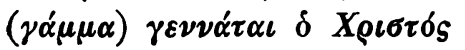

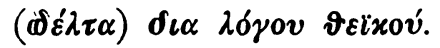

${ }^{68} R$ (esponsorium) 'P

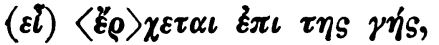

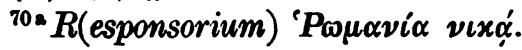

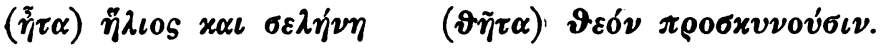

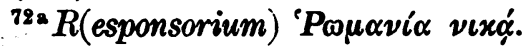

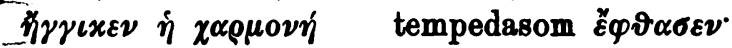

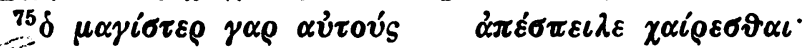

Х

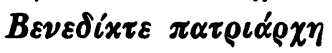

$\tau 0 \nu \delta \iota \delta \alpha ́ \sigma x \alpha \lambda o \nu \dot{\eta} \mu \omega^{\prime} \nu$

ต́s oi $\pi \alpha \downarrow \delta \varepsilon$ oi $\chi \varrho \eta \sigma \tau o l$

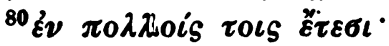

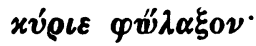

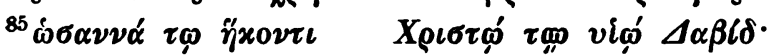

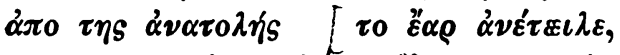

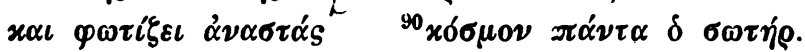

59 philacis. II. C: corr. Fabre.

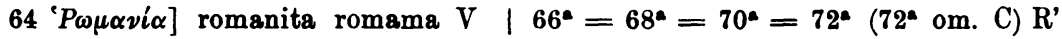
romaniß̄m (auch romanam, rom, r'o) $C^{\prime} V$; die Auflösung $R$ (esponsorium) ist sehr unsicher | 69 eichete CV.

70 wohl besser $\varphi \dot{\varepsilon} \rho \omega \nu$, da jede Langzeile hier einen Satz bildet | 72 prosci-

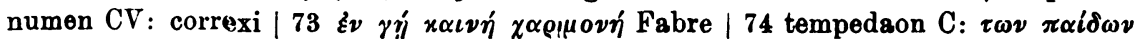

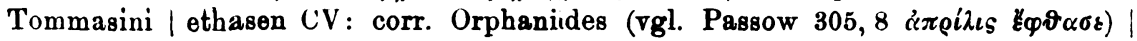
76 cherosthe CV: corr. Fabre | 78 pruginitas. I. (scr. II?) CV: corr. Fabre.

82 curie $\mathrm{CV} \mid \varphi v \hat{\lambda} \alpha \xi \circ \nu]$ ymon add. C: apron add. V| 83 oi (vor $\chi \varrho \eta \sigma \tau)$.$] isti$ $\mathrm{CV}$ (wegen christi) | 84 craigauzontes $\mathbb{W}$ : craicanzontes $\mathrm{C} \mid 88$ kै $\alpha \rho]$ Fabre: erar $\mathrm{CV} \mid 89 \varphi \omega \tau i \xi \varepsilon l]$ Tommasini: fociai C: fionai V.

NB. Die Konjekturen und Bemerkungen von Orphanides gebe ich auf Grund persönlicher Mitteilung etwa aus dem Jahre 1907. Die Deutung des Überlieferten ingicheni in v. 73 fand Th. Bolides 19012 gelegentlich einer Besprechung dieses Stïcks in Krumbachers Seminar. 
Exkurs I (vgll. S. 29).

Chronicon Paschale pag. 620 ed. Bonn.

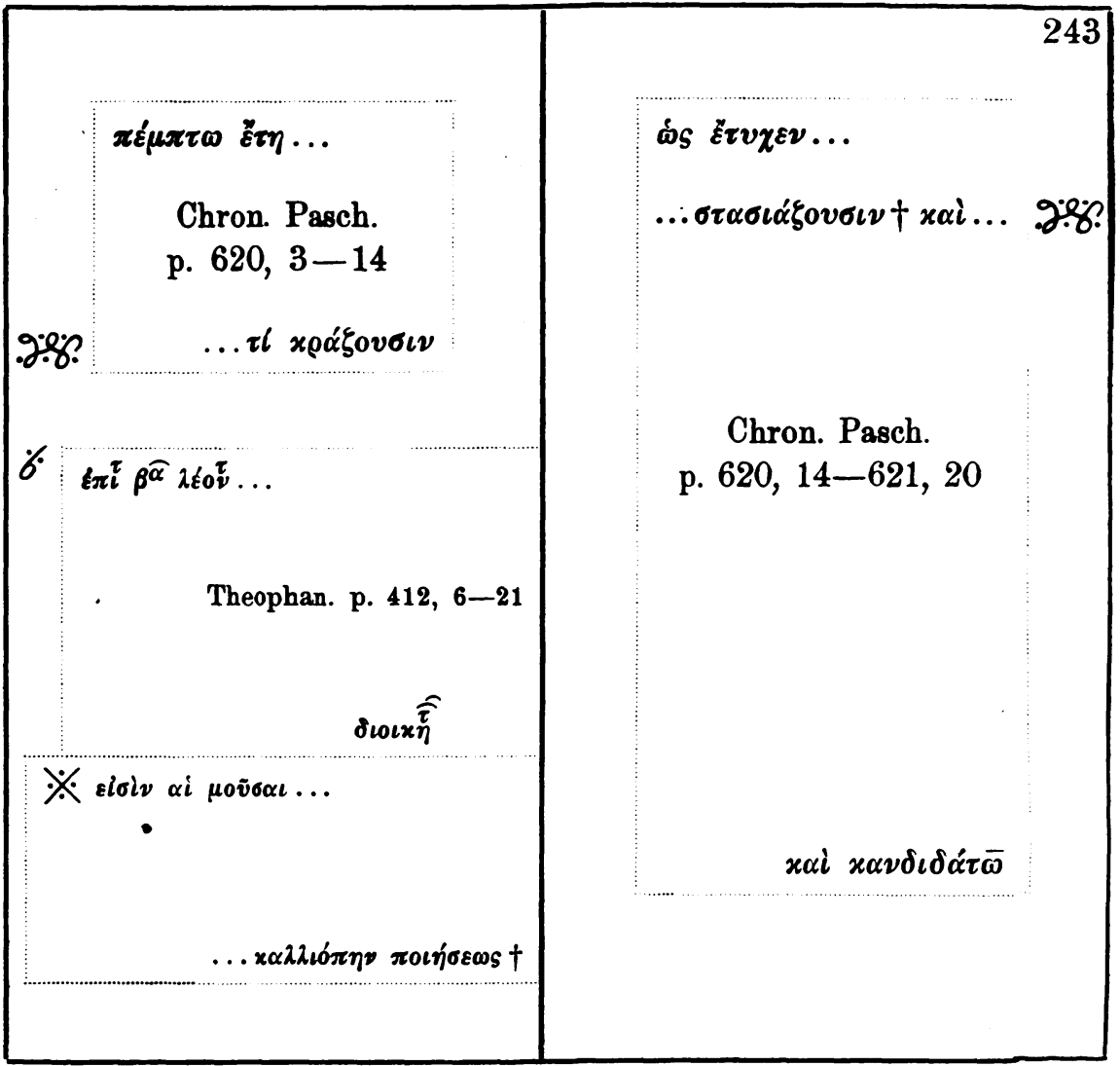

Cod. Vatic. gr. 1041 fol. $242^{\vee}-243^{r}$, schematisch, und stark verkleinert.

(Die punktierten Linien bezeichnen die Ränder der Schriftflächen.)

Der Anfang des Berichtes der 0)sterchronik über die Nikarerolte ist ungenügend ediert. Das ließe ssich leicht am Text der Ausgabe demonstrieren; aber ein Blick in die Handschrift lehrt viel mehr.

In dem Vaticanus $1941^{1}$ ), demı codex unicus der Osterchronik, schlieBt der Bericht über das Jahr 529//30 auf fol. 241' Mitte (= p. 620,2 $\chi \varrho \eta \mu \alpha ́ \tau \omega \nu$ der Bonner Ausgabe). D)er fortlaufende Text der. ersten Hand setzt erst auf fol. $243^{r}$ oben wieder ein, und zwar mit den

1) Ich besitze eine von Sansainis vorttrefflichen WeiB-Schwarzphotographien von fol. 242r-243r. Die Besorgung der Phrotographie und mehrere ergänzende Mitteilungen über die Hs danke ich Monsiigmore Giovanni Mercati. 
Worten $\dot{\omega} \varsigma$ हैं $v \chi \chi \varepsilon v(=$ p. 620,14$)$, mitten in einem Bericht über den 2. Tag der Nikarevolte (14. Jan. 532). ${ }^{1}{ }^{*}$ Die dazwischen liegende Partie ades Vaticanus enthält folgendes: 1. von erster Hand, aber mit dunklerer Tinte geschrieben, auf der oberen Hälfte von fol. $242^{\text {r die }}$

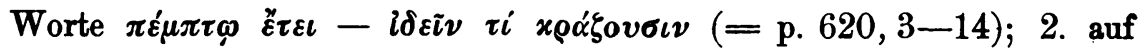
fol. $242^{\mathrm{r}}$ und den übrigen Teilen von $241^{\mathrm{r}}$ und $242^{\mathrm{r}}$ in einer ganz verschiedenen, aber vielleicht gleichzeitigen Schrift Auszüge $\tilde{\varepsilon} x$ rov

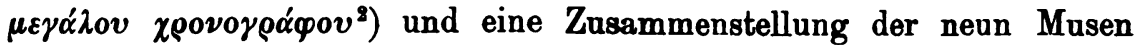
und deren Funktionen (vgl. Abbildung).

Die Frage ist nun: wie verhält sich das auf der oberen Hälfte von fol. $242^{\vee}$ überlieferte Stück zu dem fortlaufenden Text? DaB man es ihm nicht einfach vornen anheften darf, bedarf wohl keines Wortes

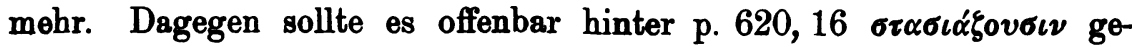
lesen werden; darauf weisen schon die respondierenden Zeichen .28? auf fol. $242^{\mathrm{r}}$ und $243^{\mathrm{r}}$ (vgl. Abbildung) ${ }^{3}$ ) Es handelt sich um einen Versuch, den ausgefallenen Anfang des Berichtes über die Nikarevolte zu ergänzen. Und woher stammt dieser Nachtrag? Aus einer vollständigen $\mathrm{Hs}$ des Chronicon Paschale keinesfalls; es fehlt ja der An-

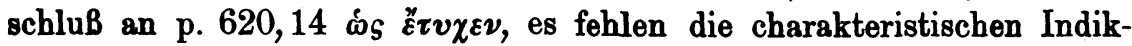
tion- und Konsulatsvermerke, und vor allem ist der Inhalt nicht einmal

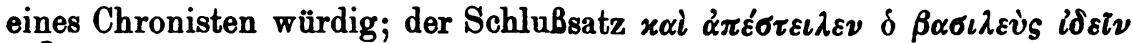

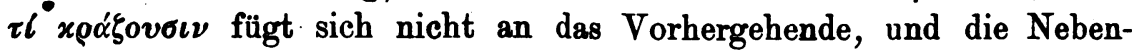

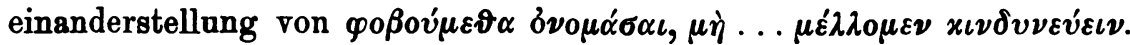

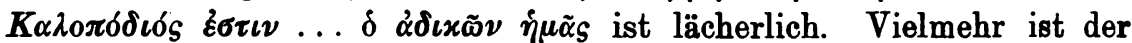

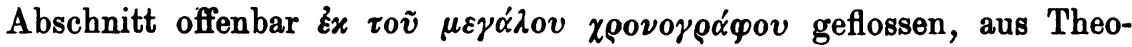
phanes (p. 181, 24-25. $32-184,2$ ), bei dem alles wörtlich, nur viel sinnvoller und ausführlicher wiederkehrt - alles bis auf jenen SchluBsatz; und dieser ist einfach eine Paraphrase der Worte p. 620,15 xai

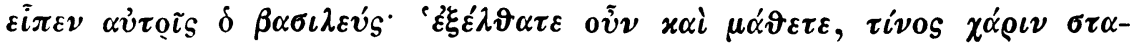

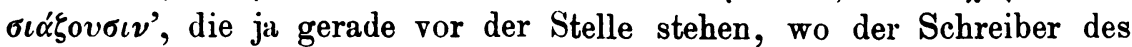

1) Geschildert ist, wie jemand dem Kaiser Vorwürfe über sein Zaudern macht; es folgt die auch beim Oxforder Malalas p. 475, $1 \mathrm{ff}$. geschilderte Szene. Quelle ist wohl der vollständige Malalas oder Johannes von Antiocheia.

2) Auf fol. $242^{v}$ steht die Schilderung des Erdbebens vom Jahre 723/4 mit

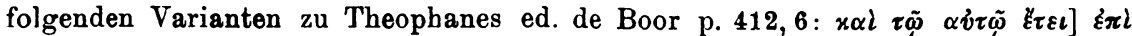

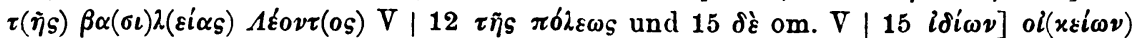
$\mathrm{V} \mid 17 \delta\left\llcorner\varepsilon \lambda \alpha^{\prime} \lambda\right.$. - $21 \delta\llcorner\iota \iota x \eta \tau \alpha \tilde{\varepsilon} s]$ ganz umgearbeitet in V. - Andere Exzerpte aus Theophanes sind zu p. 694,16 der Ostercbronik in $V$ an den Rand geschrieben (7gl. dis Arsgaben).

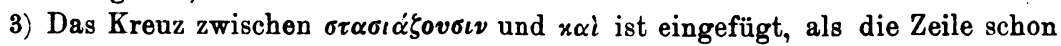
geschrieben war. 
Vaticanus seinen Nachtrag münden lassen will; er ist also offenbær sein Werk, und soll die Überleitung lherstellen. ${ }^{1}$ )

Eine Ausgabe_der Partie müBte also etwa so aussehen. edı Bonn. p. $620,1-621,3$

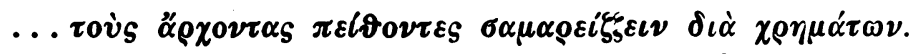

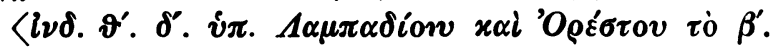
$\tau \times \eta^{\prime} \quad \delta \lambda v \mu \pi \tau \alpha^{\prime}$.

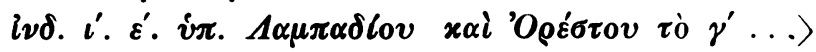

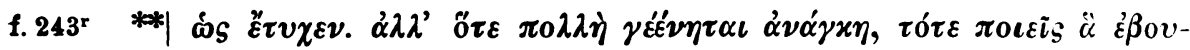

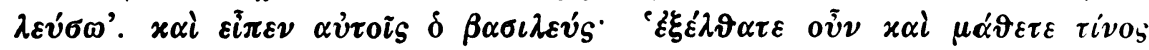

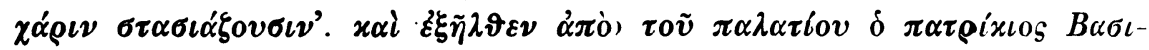

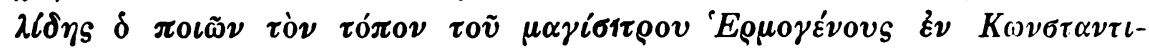

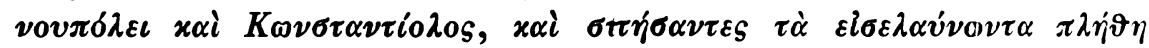

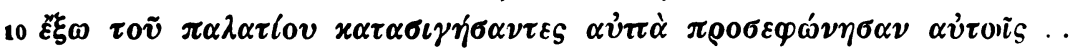

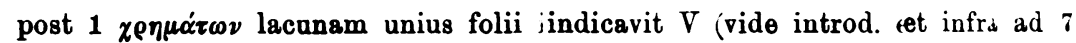
$\left.\sigma \tau \alpha \sigma \alpha^{\prime} \sigma_{0} \sigma \iota \nu\right) \mid 2-4$ partim supplevit, partim ex p. 629, 7-8 huc transposuit Rader

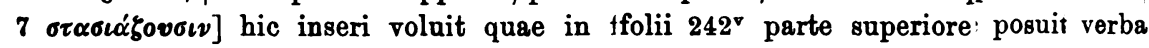
ex Theophanis chronographia p. 180 sqq. boreviata V (= p. 620, 3-1.4 ed. Bonn.):

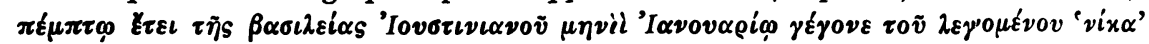

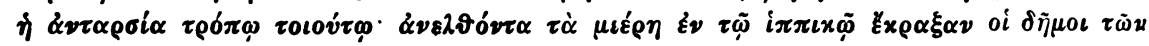

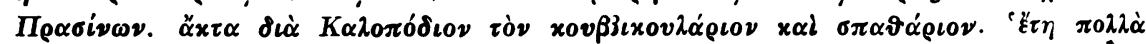

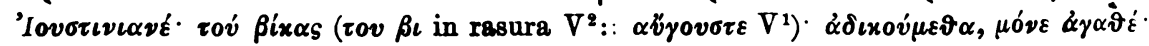

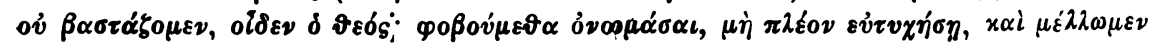

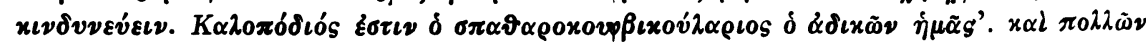

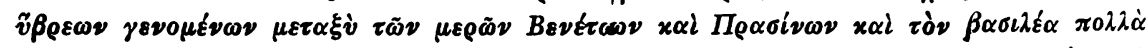

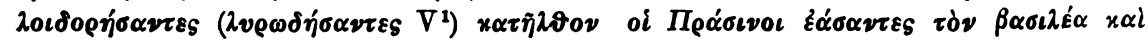

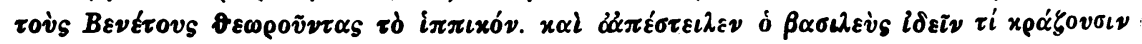

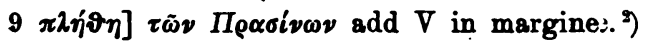

Vielleicht hilft diese Untersuclhung dazu, daß die erst jü̈ngst wieder von Eduard Schwartz (Paulyy-Wissowa III 2461, 17) dringend geforderte neue Kollation des Vaticamus 1941 endlich gemacht werde. ${ }^{3}$ )

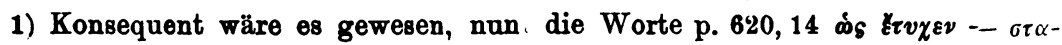

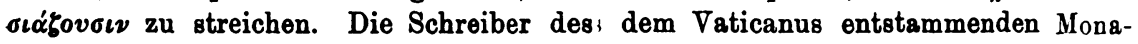
censis (auf dem die älteren Ausgaben beruihen) hat das auch wirklich getan.

2) Das ist falsch, denn es handelt sich um beide Parteien; vgl. Malalas 475,3. Diese Randnote soll offenbar die Verbindung mit dem Nachtrag festigen, in dem

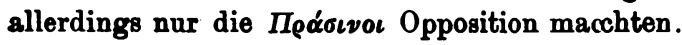

3) AuBer den im Vorangegangenen gegebenen Nachtrăgen zu der von einem unbekannten homo idoneus für Dindorf verffertigten Kollation fand ich auf fol. $\mathbf{2 4 3}^{\text {' }}$

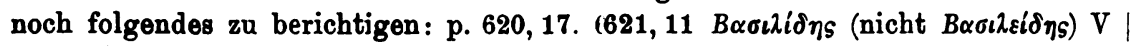

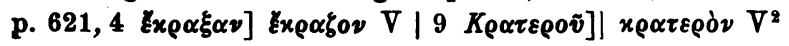


Exkurs II (vgl. S. 31).

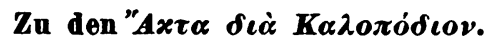

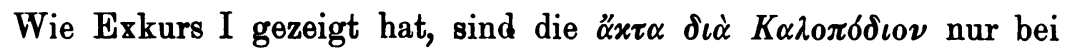
Theophanes überliefert. Dort stehen sie zwischen zwei Berichten über die Nikarevolte (Jan. 532), einem summarischen (p. 181, 24-31), der auch bei Cramer, Anecd. gr. II 112 erhalten ist, und einem ausführlichen, p. 184, 3-186, 2, der teilweise wörtlich aus Malalas stammt.

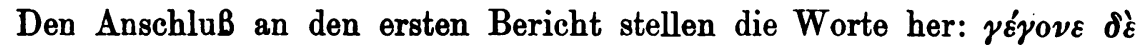

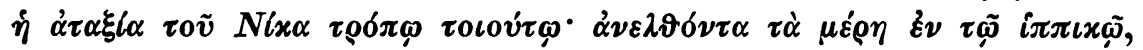

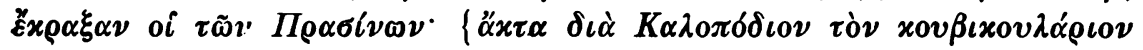

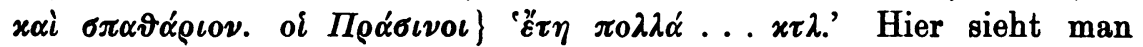
deutlich, wie ein mit Überschrift $\{\ddot{\alpha} x \tau \alpha \ldots\}$ versehenes Schriftstück sinnlos und mechanisch in den Text eingeschoben ist. Es folgt nun der Dialog und die kurze Erwähnung des Abzugs der Grünen. Nun die Fortsetzung:

Malalas 473, 5

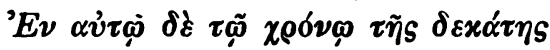

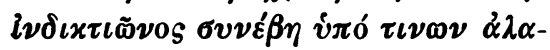

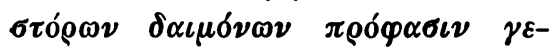
$\nu \dot{\varepsilon} \sigma \vartheta \alpha \iota \quad \tau \alpha \rho \alpha \chi \tilde{\eta} S \quad \dot{\varepsilon} \nu \quad B v \xi \alpha \nu \tau i \iota_{\varphi} E \dot{v}-$

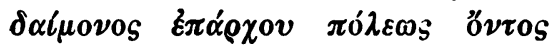

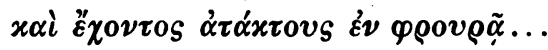

Theophanes 184, 3

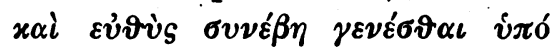

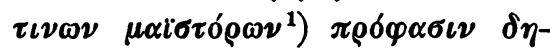

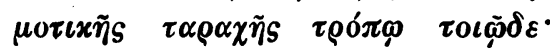

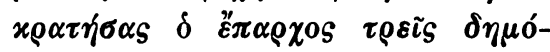
$\tau \alpha S \ldots$

Die Schilderung des Theophanes erweckt den Anschein, als schlieBe das 184, 3 ff. Erzählte zeitlich unmittelbar an den Zank im Hippodrom an. Aber das Wort, das den Zusammenhang herstellt, $\varepsilon \dot{v} \vartheta v_{s}$, ist von ihm zá eben jenem Zweck der Quelle (Malalas) hinzugefügt, die er glücklicherweise gerade hier sonst wörtlich ausschreibt. Was aber Malalas hier schildert, das ist wirklich der Ursprung der Revolte: die miBglückte Hinrichtung von Mitgliedern beider Parteien, die ihrerseits zu den vergeblichen Gnadengesuchen der Parteien am 13. Januar und damit zu der für jene Tage charakteristischen Verbindung der Grünen und Blauen führte. ${ }^{2}$ ) Theophanes ist also 184, 3 zum zweiten Mal

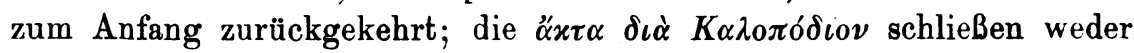
nach vorne noch nach hinten an. Ihre Datierung muB also unabhängig von der Stellung geschehen, die ihnen Theophanes anweist.

$\mathrm{DaB}$ sie in die unmittelbare Vorgeschichte des Nikaaufstandes gehören, ist jetzt sehr unwahrscheinlich. Die dominierende Note, Justi-

1) Verlesen aus A^ACTOPWN, wie Bury B. Z. VI (1897) 508 erkannt hat. Ich traue den Irrtum dem Theophanes zu.

2) Vgl. Bury, The Nika Riot, Journal of Hellenic Studies 17 (1897) 92-119.

Byzant Zeitschrift XXI 1 n. 2 
nians MiBgunst gegen die Grümen ${ }^{1}$ ), paßt überall besser hin als in jene Tage, in denen sich die Reegierung gerade dadurch schadete, daB sie ausnahmsweise unparteilich vorging. Positives kann ich jedoch

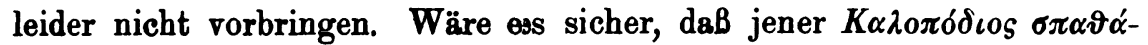

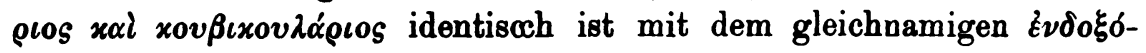

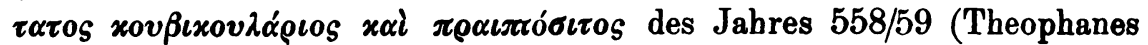
a. 6051 p. 233, 8 nach Malalas 490,9), so hätte man wenigstens einen Terminus ante quem; denn dass Amt des praepositus ist das höhere.

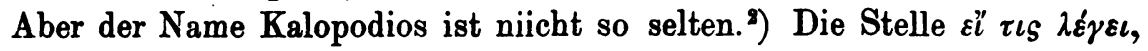

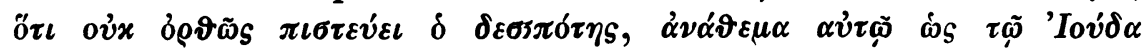
(Z. $25 \mathrm{f}$.) spielt an auf $\mathrm{Zweifel}$ an der Orthodoxie des Kaisers; man erinnert sich, daB Justinian kurzz vor seinem Tod dem Aphthartodoketismus verfiel. Aber die ganze sStelle ist rätselhaft, besonders im Mund der Opposition. Aus den zahlrreichen Krawallen unter Justinian, von denen wir wissen, einen auszuwï̈hlen, etwa den für die Grünen besonders verhängnisvollen vom Nov.. 561 (Theophanes a. 6054, p. 235, 29), wäre Willkür.

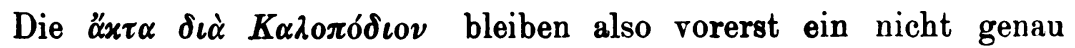
datiertes Stimmungsbild aus den Streitigkeiten der Parteien unter Justinian, auch so noch einzigartig und unschätzbar. Es sind Klagen der Grünen über Mißhandlung seitens der Regierung, und Zurückweisung der Klagen durch den Mandatorr (Herold) des Kaisers. Anfangs spielt der Name eines den Grünen bessonders verhabten Beamten hinein, des Kalopodios; gegen Ende mischen sich auch die Blauen in den Zank. Die Unterhaltung wird von seiiten der Grünen durchweg in ruhigem Ton geführt, obwohl die Antworten des Mandators sehr bald unparlamentarisch klingen. Die Grüncen verstecken ihre Wut unter scheinbarer Resignation, die sich zeitweise mit grimmigem Hohn und dunkeln Drohungen mischt. SchlieBlich reiBt ihnen die Geduld, und mit einen Fluch auf die, die zurückbleibem, verlassen sie das Hippodrom.

Die Einzelinterpretation sclharf durchzuführen halte ich angesichts der hochgradigen Verderbnis dess Textes für aussichtslos. Selbst darauf mußte ich verzichten, die mir unverständlichen Stellen durch Kreuze zu bezeichnen; denn die Haupträtsel liegen nicht in einzelnen $\mathrm{W}_{\text {ör }}$ tern, sondern in der Verbindumg der Sätze. So habe ich mich damit

1) Prokop. Anecd. 7, 1. 9, 33. Theoph. a. 6061 p. 243, 7, durch die die Vermutung des Euagrios 4, 32, der diesse Feindschaft erst vom Nikaaufstand datiert, widerlegt wird.

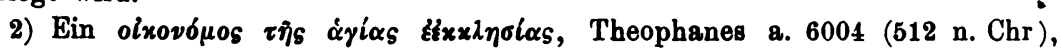

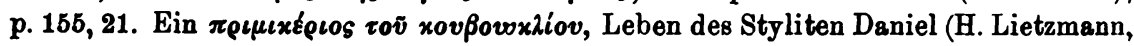
Byzantin. Legenden, 1911, 23. 100), : a. ca. 466. 
begnügt, die Metrik, die gewiß andere ebenso überrascht wie mich ${ }^{1}$ ), so gut es ging, klarzulegen.

Noch ein paar Worte über eine sprachliche Erscheinung. Die Parteien reden von sich regelmäBig im Singular; wo sie angeredet werden, kommen beide Numeri vor. Das könnte zu der Annahme verführen, sie sprächen durch einen Wortführer. Dem würde aber nicht nur die gleiche Erscheinung in mehreren anderen Massenakklamationen ${ }^{8}$ ) widersprechen, sondern vor allem die Überlieferung über den Vortrag solcher Akklamationen, die das Eigentümliche, ja Unheimliche des im. provisierten Zusammensprechens so vieler Menschen bezeugt ${ }^{3}$ ). Eine bedeutsame Parallele für den Gebrauch des Singulars bietet der Chor des antiken Dramas.

Nachtrag zu der Textsammlung. Bei Malalas 361, 19 (danach Chronic. Pasch. 588, 13 und Theophanes a. 5937). wird folgende, vielleicht metrische Akklamation der Byzantiner auf den Stadtpräfekten Kyros üborliefert, der sich unter Theodosios II durch Bauten um die Stadt verdient gemacht hatte:

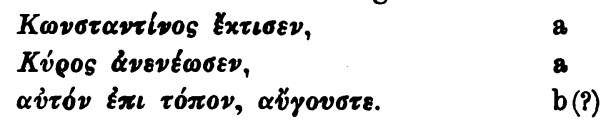

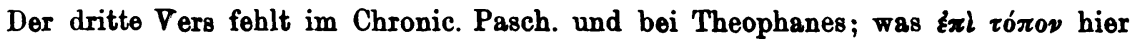
heißt, ist nicht klar. Eine andere Akklamation auf Kyros bei den Script. Orig. Const. 252, 14 Preger.

Berlin.

Paul Maas.

1) Angesichts dieses Fundes, sowie des für die Geschichte der byzantinischen Metrik nicht minder bedeutsamen der ältesten gleichzeiligen Poesie (B. Z. XVIII 317 ff.), wird wan verzeihlich finden, daB ich zogere, meine seit Jahren fertiggestellte byzantinische Metrik zu drucken.

2) Oben III 1, 5. 7; Mansi VIII 1058; Theophanes a. 6071, p. 249, 25; Const. Porph. de cser. 326, 1.6 etc.

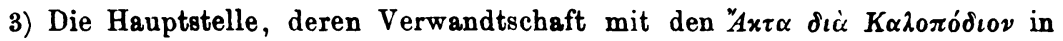
die Augen springt, möge den Rest der Seite füllen. Cassius Dion berichtet als

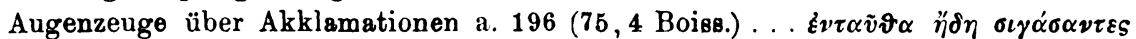

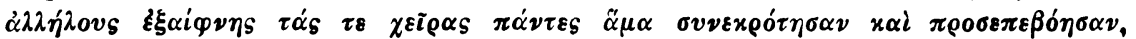

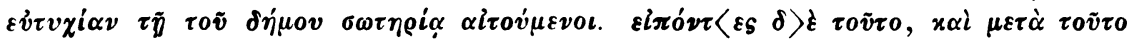

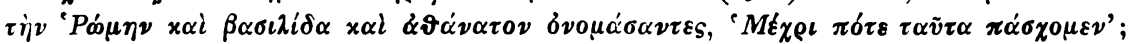

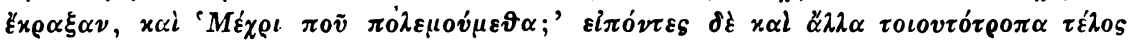

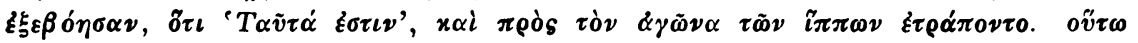

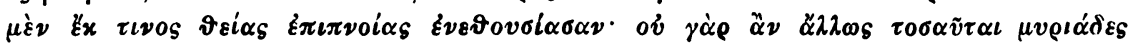

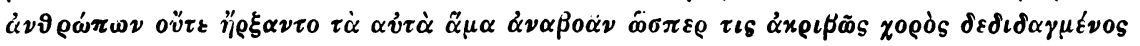

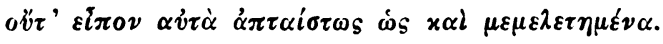

